\title{
Mecânica estatística de sistemas complexos
}

Statistical Mechanics for Complex Systems

\author{
Constantino Tsallis ${ }^{* 1,2,30}$ \\ ${ }^{1}$ Centro Brasileiro de Pesquisas Físicas, Instituto Nacional de Ciência e Tecnologia de Sistemas Complexos \\ Rio de Janeiro, RJ, Brasil. \\ ${ }^{2}$ Santa Fe Institute, New Mexico, USA. \\ ${ }^{3}$ Complexity Science Hub Vienna, Vienna, Austria.
}

Recebido em 12 de setembro de 2020. Aceito em 19 de outubro de 2020.

\begin{abstract}
A magnífica mecânica estatística de Boltzmann-Gibbs, amálgama de primeiros princípios e teoria de probabilidades, constitui um dos pilares da física teórica contemporânea. Entretanto, ela não se aplica a grande número dos sistemas ditos complexos, caracterizados essencialmente por um forte emaranhamento espaço-temporal de seus elementos. Revisamos aqui a proposta de generalização chamada mecânica estatística não extensiva, que emergiu em 1988. Ela está baseada em entropias não aditivas (com índice $q \neq 1$ ), em contraste com a entropia de Boltzmann-Gibbs-von Neumann-Shannon, que é aditiva (com índice $q=1$ ). Sua fundamentação básica, assim como aplicações selecionadas em física e fora dela, são brevemente descritas.

Palavras-chave: Mecânica estatística não extensiva, entropias não aditivas, sistemas complexos, sistemas dinâmicos não lineares.
\end{abstract}

The magnificent Boltzmann-Gibbs statistical mechanics, amalgam of first principles and theory of probabilities, constitutes one of the pillars of contemporary theoretical physics. However, it does not apply to a wide number of the so called complex systems, characterized essentially by a strong space-time entanglement of its elements. We tutorially review here the proposal for its generalization, referred to as nonextensive statistical mechanics, which emerged in 1988. It is based on nonadditive entropies (with index $q \neq 1$ ), in contrast with the Boltzmann-Gibbsvon Neumann-Shannon entropy, which is additive (with index $q=1$ ). Its basic foundations, as well as selected applications in physics and elsewhere, are briefly described.

Keywords: Nonextensive statistical mechanics, nonadditive entropies, complex systems, nonlinear dynamical systems.

Si l'action n'a quelque splendeur de liberté, elle n'a point de grâce ni d'honneur. Montaigne

\section{Introdução}

$\mathrm{Na}$ física teórica contemporânea, cinco são as disciplinas obrigatórias em qualquer curso avançado de Física ao redor do mundo: mecânica clássica ou Newtoniana, sua generalização para velocidades próximas à da luz, qual seja a mecânica relativista restrita de Einstein, assim como sua generalização para massas pequenas, qual seja a mecânica quântica, o eletromagnetismo de Maxwell, e finalmente a mecânica estatística de Boltzmann-Gibbs (MEBG), incluindo, para sistemas com grande número de elementos, sua conexão com a Termodinâmica clássica. Lembremos que as equações centrais da mecânica clássica são $\vec{F}=m \vec{a}$ e $E=$ $p^{2} / 2 m$. As da teoria da relatividade são $\vec{F}=\frac{d \vec{p}}{d t} \mathrm{e}$ $E=\sqrt{m^{2} c^{4}+p^{2} c^{2}}$. A equação central da mecânica

\footnotetext{
*Endereço de correspondência: tsallis@cbpf.br
}

quântica é a de Schrodinger, e as do eletromagnetismo as de Maxwell. Finalmente, as equações centrais da teoria de Boltzmann-Gibbs (BG) são $p_{i} \propto e^{-E_{i} / k_{B} T} \mathrm{e}$ $S=k_{B} \ln W$, e a da Termodinâmica é $\frac{1}{T}=\frac{\partial S}{\partial U}$. Não temos definido matematicamente estas varias grandezas aqui por considerar isto desnecessário para o leitor típico deste artigo. Mencionemos entretanto, por oportuno, que até aparecerem a teoria da relatividade restrita e a mecânica quântica no inicio do século XX, usava-se simplesmente a expressão mecânica, pois só existia a de Newton. Analogamente, até 1988, quando foi proposta a mecânica estatística não extensiva (MENE) [1] (ver detalhes em [2, 3]), usava-se simplesmente a expressão mecânica estatística, pois só existia a de BG. A situação tem hoje consideravelmente evoluído, como focalizaremos precisamente neste artigo. Desde o ponto de vista epistemológico, veremos que a mecânica estatística não extensiva generaliza a de BG de modo semelhante a como a relatividade restrita e a mecânica quântica contêm a mecânica de Newton; ou ainda como a relatividade geral de Einstein contém a restrita. Na Fig. 1 ilustramos esquematicamente as mais importantes conexões que nos levam das teorias 


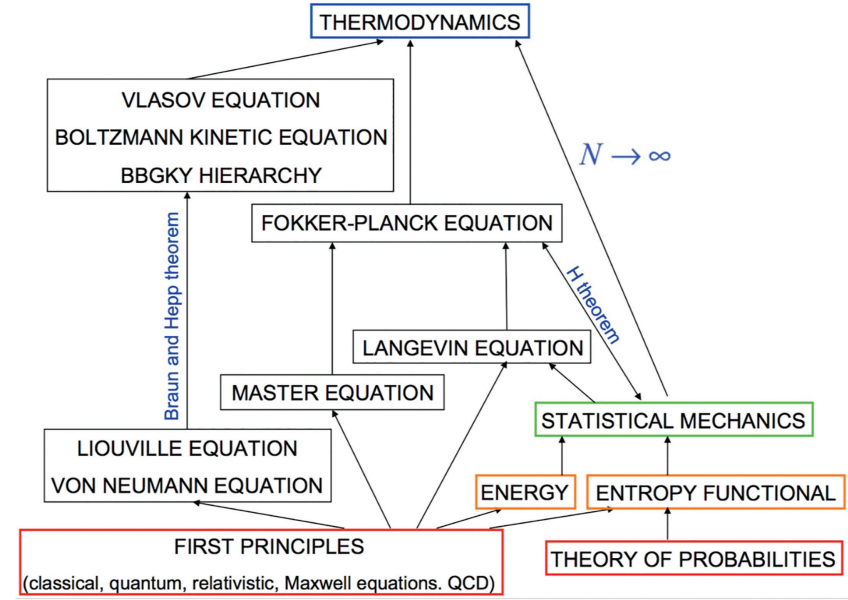

Figura 1: Descrições microscópica (de primeiros princípios da eletro-mecânica), mesoscópicas e macroscópica (termodinâmica) da natureza em física teórica contemporânea incluindo varias, obviamente não todas, equações relevantes. A energia emerge da eletro-mecânica como uma constante de movimento. O funcional entrópico emerge de primeiros princípios eletro-mecânicos como uma função adequada das probabilidades diretamente relacionadas às trajetórias no espaço de fases, ou no espaço de Hilbert, ou no de Fock, e às correlações ali envolvidas. O teorema de Braun e Hepp que é mencionado em uma das ligações é aquele provado em [4]. Somente apresentamos aqui um esquema básico: outros ramos e conexões existem que não estão indicados neste diagrama (veja por exemplo [5]).

ditas de primeiros princípios (descrição microscópica) até a Termodinâmica (descrição macroscópica), através de diversas aproximações intermediarias (descrição mesoscópica).

Vejamos agora: quais são as características que fazem que um sistema obedeça as leis da mecânica estatística de $\mathrm{BG}(q=1$, como explicitaremos em breve), e quais aquelas que o levam a obedecer as leis da MENE $(q \neq 1)$ ? De uma maneira intuitiva e simplificada podemos dizer que um sistema (incluindo sua circunstância) pode ser simples ou complexo, correspondendo tipicamente a $q=1$ e a $q \neq 1$, respectivamente. Mas e em que consiste, neste contexto, a complexidade? (área de estudo de uma ciência à qual Murray Gell-Mann gostava de se referir como plectics, do grego plektós, tecido). Como a beleza, a complexidade é difícil de definir porém relativamente fácil de identificar [6]. Assim, os sistemas complexos tipicamente possuem relevantes características não lineares. Esta não linearidade pode ser dinâmica, estrutural, e outras que ilustraremos gradualmente ao longo da presente revisão. Podemos adiantar dizendo que correlações espaço-temporais entre os graus de liberdade do sistema decaindo rapidamente (e.g., exponencialmente no regime assintótico) apontam para a MEBG $(q=1)$, enquanto se caem lentamente (e.g., como leis de potências no regime assintótico) apontam frequentemente para MENE $(q \neq 1)$. Uma ressalva se impõe: enquanto existe essencialmente uma única maneira de ser simples, existem infinitas maneiras de ser complexo. Assim, num dado contexto, existe uma única maneira de ser linear e infinitas maneiras de ser não linear. Ou ainda, nas palavras de Liev Tolstoi em Anna Karénina: Todas as famílias felizes se parecem, cada família infeliz é infeliz à sua maneira. Trocando em miúdos para nosso contexto: a mecânica estatística de BG é única (e, portanto, paradigmática); as não Boltzmannianas são infinitas, sendo a MENE apenas uma destas. Entretanto, por razões que até agora permanecem ilegíveis - até intrigantes -, a MENE, suas $q$-exponenciais e suas $q$-Gaussianas (que definiremos em breve, e que, para $q=1$, reproduzem respectivamente as exponenciais e Gaussianas que constituem as inconfundíveis impressões digitais da MEBG) emergem, como veremos, com surpreendente frequência nos mais diversos sistemas naturais, artificiais e sociais.

Fechando esta introdução, é oportuno fazer o comentário que segue. Em qualquer bom livro de mecânica quântica tem 5 sistemas que são detalhadamente analisados. Estes são: (1) uma partícula em um poço quadrado, (2) um oscilador harmônico, (3) um rotor rígido, (4) uma partícula com spin $1 / 2$ na presença de campo magnético externo, e (5) um átomo de Hidrogênio não ionizado. Em qualquer bom livro de MEBG são detalhadamente analisadas as propriedades térmicas básicas (e.g., a equação de estado, o calor específico) dos primeiros quatro,... mas não do quinto! Por que? Mais ainda: Por que é raramente explicitada esta intrigante ausência? O motivo é, entretanto, simples: a interação Coulombiana elétron-próton é de longo alcance, portanto a função de partição de $B G$ diverge, o que inviabiliza o cálculo de qualquer propriedade termoestatística no equilíbrio térmico []$^{1}$ De fato, se não supormos que este átomo se encontra confinado em algum volume finito, ele simplesmente carece de equilíbrio térmico a qualquer temperatura diferente de zero. Eis uma boa razão, entre muitas outras, para generalizar a MEBG! (no quadro de um formalismo teórico mais flexível, talvez seja possível discutir utilmente sistemas como esse em termos de estados quasi-estacionários não-Boltzmannianos).

\footnotetext{
${ }^{1}$ Gibbs escreve: In treating of the canonical distribution, we shall always suppose the multiple integral in Equation (92) [a função de partição, como é chamada hoje] to have a finite value, as otherwise the coefficient of probability vanishes, and the law of distribution becomes illusory. This will exclude certain cases, but not such apparently, as will affect the value of our results with respect to their bearing on thermodynamics. It will exclude, for instance, cases in which the system or parts of it can be distributed in unlimited space [...]. It also excludes many cases in which the energy can decrease without limit, as when the system contains material points which attract one another inversely as the squares of their distances. [...]. For the purposes of a general discussion, it is sufficient to call attention to the assumption implicitly involved in the formula (92).
} 


\section{Entropias Não Aditivas e Termodinâmica}

Um funcional entrópico $S\left(\left\{p_{i}\right\}\right)\left(\operatorname{com} \sum_{i=1}^{W} p_{i}=1\right)$ (ou simplemente entropia) é dito aditivo [8] se, para dois sistemas $A$ e $B$ probabilísticamente independentes (i.e., $\left.p_{i j}^{A+B}=p_{i}^{A} p_{j}^{B}, \forall(i, j)\right), S(A+B)=S(A)+S(B)$. Caso contrario, a entropia é dita não aditiva.

A entropia de BG, base da MEBG, é definida como segue:

$$
S_{B G}\left(\left\{p_{i}\right\}\right)=k \sum_{i=1}^{W} p_{i} \ln \frac{1}{p_{i}}
$$

onde $k$ é uma constante convencional positiva (usualmente $k=k_{B}$ em física, e $k=1$ em teoria da informação). Para equiprobabilidade, $S_{B G}$ atinge o valor

$$
S_{B G}=k \ln W .
$$

É trivial verificar que $S_{B G}$ dada pela Eq. (1) é aditiva. Existe de fato uma única generalização de $S_{B G}$, a entropia de Renyi $S_{q}^{R}=k \frac{\ln \sum_{i=1}^{W} p_{i}^{q}}{1-q}$, que também é aditiva, $\forall q$. Ela é útil para caracterizar estruturas geométricas tais como multifractais, e de modo geral na teoria da informação, mas não necessariamente em termodinâmica propriamente dita pois carece de algumas propriedades matemáticas que parecem ser imprescindíveis para este fim.

Podemos generalizar $S_{B G}$ de outra maneira, qual seja 1

$$
\begin{aligned}
S_{q}\left(\left\{p_{i}\right\}\right) & =k \frac{1-\sum_{i=1}^{W} p_{i}^{q}}{q-1}=k \sum_{i=1}^{W} p_{i} \ln _{q} \frac{1}{p_{i}} \\
& =-k \sum_{i=1}^{W} p_{i}^{q} \ln _{q} p_{i} \\
& =-k \sum_{i=1}^{W} p_{i} \ln _{2-q} p_{i},\left(q \in \mathcal{R} ; S_{1}=S_{B G}\right),
\end{aligned}
$$

onde

$$
\ln _{q} z \equiv \frac{z^{1-q}-1}{1-q},\left(\ln _{1} z=\ln z\right) .
$$

Para equiprobabilidade, $S_{q}$ atinge o valor

$$
S_{q}=k \ln _{q} W .
$$

Para variáveis contínuas $S_{q}$ toma a forma

$$
S_{q}(\{p(x)\})=k \frac{1-\int d x[p(x)]^{q}}{q-1}=k \int d x p(x) \ln _{q} \frac{1}{p(x)},
$$

e, em mecânica quântica, toma a forma

$$
S_{q}(\rho)=k \operatorname{Tr}\left[\rho \ln _{q}(1 / \rho)\right],
$$

onde $\rho$ é a matriz densidade.
Podemos facilmente verificar que

$$
\frac{S_{q}(A+B)}{k}=\frac{S_{q}(A)}{k}+\frac{S_{q}(B)}{k}+(1-q) \frac{S_{q}(A)}{k} \frac{S_{q}(B)}{k},
$$

ou ainda

$$
S_{q}(A+B)=S_{q}(A)+S_{q}(B)+\frac{1-q}{k} S_{q}(A) S_{q}(B) .
$$

Vemos assim que, para $q \neq 1, S_{q}$ é não aditiv ${ }^{2}$ Outrossim, ela possui propriedades (tais como, por exemplo, concavidade e estabilidade de Lesche para todo $q>0$, convexidade para todo $q<0$, composabilidade para todo $q$, unicidade nos axiomas $q$-generalizados de Shannon [9] e de Khinchin [10], admissibilidade nos axiomas de Shore-Johnson [11], entre outras [2, 3], 12]), que conduzem à sua essencial utilidade para formular a MENE. Vemos que, para $\frac{1-q}{k} \rightarrow 0$ (portanto para $1 / k \rightarrow 0$ para $q$ fixo, ou para $q \rightarrow 1$ para $k$ fixo), a relação (9) recupera a aditividade de $S_{B G}$. Este fato é análogo com a conhecida composição de velocidades $\left(v_{12}=\frac{v_{1}+v_{2}}{1+\left(v_{1} v_{2}\right) / c^{2}}\right)$ em teoria da relatividade: ela é não linear, mas no limite onde a velocidade da luz $c \rightarrow \infty$ recupera a composição Galileana (linear) de velocidades $\left(v_{12}=v_{1}+v_{2}\right)$ que constitui uma das marcas da mecânica Newtoniana. Podemos nos perguntar: por que, na sua generalização da teoria de Newton, Einstein admitiu violar a simpática e intuitiva fórmula de Galileo? Resposta: era um preço pequeno a pagar para atingir um objetivo muito maior, qual seja a invariância de Lorentz da teoria, o que permitia unificar a mecânica com o eletromagnetismo de Maxwell. Analogamente, admitiremos violar a simples aditividade de $S_{B G}$ para atingir um objetivo muito maior, qual seja a preservação da termodinâmica clássica para qualquer valor de $q$. Este ponto constitui um dos pilares da MENE, e é o que discutiremos a seguir.

Imaginemos por simplicidade que temos equiprobabilidade no sistema, portanto $p_{i}=1 / W, \forall i$. Se as $N$ variáveis aleatórias do sistema são aproximadamente independentes, temos que $W(N) \sim \mu^{N} \operatorname{com} \mu>1$ (por exemplo, o número total de configurações de $N$ moedas não viciadas independentes é $W(N)=2^{N}$ ). Consequentemente temos que $S_{B G}(N)=k \ln W(N) \propto N$, portanto a entropia total é extensiva como exigido pela termodinâmica clássica!

Mas se as $N$ variáveis do sistema estão fortemente correlacionadas, acontece frequentemente que $W(N) \sim N^{\rho}$ com $\rho>0$. Neste caso, não devemos usar a expressão de $\mathrm{BG}$ pois teríamos $S_{B G}(N)=k \ln W(N) \propto \ln N$, que não é extensiva, violando portanto a termodinâmica clássica que, com sua estrutura de transformações de Legendre, impõe a extensividade da entropia total em todos os casos (ver [13] e suas referências). Em contraste, podemos facilmente verificar que, para $q=1-1 / \rho$, $S_{q}(N)=k \ln _{q} W(N) \propto N$, sendo assim extensiva, satisfazendo portanto a termodinâmica clássica! Neste caso,

\footnotetext{
$\overline{2}$ Existe hoje na literatura grande número de entropias não aditivas, mas a que está diretamente associada à MENE é $S_{q}$.
} 
vemos que sacrificar a aditividade da entropia $S_{B G}$ nos permite atingir o objetivo superior de satisfazer a estrutura matemática das transformações de Legendre da Termodinâmica. Esta propriedade constitui de fato a pedra angular da MENE! (ver [14]).

\section{Otimizando $S_{q}$ com Vínculos Canônicos Simples}

Seguindo as linhas tradicionais de Boltzmann e Gibbs, ilustremos o ensemble canônico baseado na entropia (3). Mais precisamente, otimizando

$$
S_{2-q}(\{p(x)\})=-k \int d x p(x) \ln _{2-q} \frac{1}{p(x)}
$$

com os vínculos

$$
\int d x p(x)=1
$$

$\mathrm{e}$

$$
\int d x E(x) p(x)=U
$$

obtemos

$$
p_{q}(x)=\frac{e_{q}^{-\beta_{q} E(x)}}{\int d y e_{q}^{-\beta_{q} E(y)}},
$$

onde a função $q$-exponencial $e_{q}^{z} \equiv[1+(1-q) z]_{+}^{1 /(1-q)}$ $\left(e_{1}^{z}=e^{z}\right)$ é a função inversa de $\ln _{q} z ;[Z]_{+}$é igual a $Z$ se $Z>0$, e é igual a zero caso contrario; a temperatura inversa efetiva $\beta_{q}$ está relacionada com $U$ através das Eqs. 11) e 12); naturalmente, para $q=1$, recuperamos o célebre fator de BG; para $q>1$ e $z<0$ temos um decaimento assintótico em lei de potência, $e_{q}^{z} \sim 1 /(-z)^{1 /(q-1)}$; para $q<1$ e $z<0$ temos um corte para $z<1 /(1-q)$. O fato de termos formalmente otimizado $S_{2-q}$ no lugar de $S_{q}$ é um procedimento isomorfo e simplifica os cálculos (ver detalhes em [15]17]).

Se usamos $E(x)=a x^{2}(a>0)$ no vinculo 12 , obtemos a função $q$-Gaussiana

$$
p_{q}(x)=\frac{e_{q}^{-\beta_{q} a x^{2}}}{\int d y e_{q}^{-\beta_{q} a y^{2}}} .
$$

Como ilustraremos em breve, as distribuições 13 e 14 emergem na natureza e alhures com uma frequência impressionante, que teria sido obviamente difícil de prever nos primórdios da proposta publicada em 1988 (quem diria em 1985, quando foi de fato formulada). A única maneira de entender a razão pela qual tamanha universalidade é observada parece ser com base em estruturas do tipo do Teorema do Limite Central (TLC) e a Teoria dos Grandes Desvios (TGD) na teoria de probabilidades. Por este motivo, faremos na próxima sessão uma breve revisão destas estruturas.

\section{4. q-generalizando o Teorema do Limite Central e a Teoria dos Grandes Desvios}

Observamos na natureza um número imenso de situações envolvendo Gaussianas (distribuições normais) ou distribuições muito próximas a elas: penetração de um perfume num ambiente fechado, difusão de açúcar em uma xícara de café, distribuição de pesos e alturas dos indivíduos de uma dada especie de mamíferos, notas obtidas por grande número de estudantes em provas coletivas, para somente citar uns poucos exemplos. Acontece que a soma, ou a media aritmética, de muitas variáveis estocásticas independentes (ou quase) se aproxima inexoravelmente de uma Gaussiana quando o número destas variáveis cresce indefinidamente. Mais precisamente, isto acontece quando a variância das distribuições envolvidas é finita; caso ela divirja, os atratores no espaço das distribuições não mais são Gaussianas mas distribuições de Lévy (ou distribuições $\alpha$-estáveis), que assintóticamente decaem como leis de potência; no limite $\alpha \rightarrow 2$ as distribuições de Lévy recuperam as Gaussianas, e para $\alpha=1$ elas reproduzem as distribuições de Cauchy-Lorentz. É oportuno salientar neste ponto que, na natureza, são muito frequentes tanto as Gaussianas quanto distribuições empíricas que, ao longo de algumas décadas logarítmicas, exibem um comportamento em lei de potência (i.e., retas na representação log-log). Durante muitos anos estas últimas foram levianamente associadas a distribuições de Lévy, mas a realidade é que pouquíssimas delas, se algumas, foram indubitavelmente mostradas de seguir a forma de Lévy na parte central da distribuição empírica, em particular o fato que, em representação log-log, elas necessariamente exibem, para $1<\alpha<2$, um ponto de inflexão (ponto que inexiste nas $q$-exponenciais e nas $q$-Gaussianas) [18]. Existem, de fato, infinitas distribuições diferentes das distribuições de Lévy que assintóticamente seguem leis de potência. Dentre elas, as $q$-exponenciais, as $q$-Gaussianas [19], e as distribuições $(q, \alpha)$-estáveis [20].

Quando a forte hipótese de (quase) independência é dispensada e é substituída por uma classe específica de correlações (usualmente referidas como $q$-independência), as somas acima mencionadas convergem para distribuições $q$-Gaussianas, como provado em [19]. Esta propriedade é usualmente referida como o $q$-Teorema do Limite Central. Este teorema evidencia de fato condições suficientes, mas não necessárias, para termos $q$-Gaussianas como atratores no espaço das distribuições. Um teorema mais completo é muito desejável mas infelizmente não foi ainda estabelecido. Entretanto, é ao longo deste caminho que podemos intuir a razão de existirem tantos sistemas exibindo distribuições $q$-Gausianas.

A Teoria dos Grandes Desvios focaliza a 'velocidade' à qual um atrator Gaussiano é atingido ao crescer o número $N$ de variáveis. $\mathrm{Na}$ ausência de correlações 
importantes, esta velocidade é caracterizada por uma aproximação exponencial do tipo $P(N) \propto e^{-r_{1} N}$, onde a "rate function" $r_{1}>0$ é proporcional à entropia relativa de BG por partícula. Assim, $r_{1} N$ corresponde a uma entropia total extensiva. Esta probabilidade $P(N)$ é a contrapartida matemática 21 do fator de BG $p_{B G}(\mathcal{H}) \propto$ $e^{-\beta \mathcal{H}}$, onde o Hamiltoniano $\mathcal{H}(N)$ de um sistema de $N$ partículas com interações de curto alcance é extensivo, i.e., proporcional a $N$.

Quando, devido a importantes correlações, o atrator no sentido do TLC é uma $Q$-Gaussiana, seria desejável que $P(N) \propto e_{q}^{-r_{q} N}$ onde $q=f(Q)$ é uma função satisfazendo $f(1)=1$, e onde o "rate function" generalizado $r_{q}$ represente ainda uma entropia relativa generalizada por partícula. Assim a entropia total seria ainda extensiva, mesmo não mais sendo aditiva para $q \neq 1$. Esta conjectura parece ser satisfeita em varias exemplos estudados na literatura [22 25]. Desnecessário dizer que uma prova geral deste comportamento é fortemente desejável, pois ela constituiria a contrapartida matemática do fator $p_{q} \propto e_{q}^{-\beta_{q} \mathcal{H}}$, onde $\mathcal{H}(N)$ é o Hamiltoniano de um sistema de $N$ partículas com interações de longo alcance, por exemplo gravitação Newtoniana ou plasmas não neutros. Este longo alcance implica num Hamiltoniano superextensivo, mas, levando em conta que $\beta_{q}$ consistentemente cessa de ser intensivo, o produto $\beta_{q} \mathcal{H}$ preserva a extensividade que ele trivialmente satisfazia no caso de curto alcance.

\section{Algumas Aplicações}

A MENE, ocasionalmente referida como $q$-estatística por simplicidade, conta atualmente com grande número de predições, verificações, aplicações em geral. Uma Bibliografia regularmente atualizada pode ser consultada em [26], assim como artigos teóricos, experimentais, observacionais e computacionais selecionados. Mencionaremos brevemente aqui alguns deles que ilustram o atual status da proposta, feita mais de três décadas atrás, de generalização da magnífica mecânica estatística de BG.

\subsection{Mapa logístico no limiar do caos}

O mapa logístico é definido como segue:

$$
x_{t+1}=1-a x_{t}^{2}\left(a \in(0,2) ; x_{t} \in[-1,1] ; t=0,1,2, \ldots\right)
$$

Ele é o mais simples e paradigmático dos mapas dissipativos. Para $a=2$ ele exibe caos forte (com expoente de Lyapunov $\lambda_{1}(2)=\ln 2$, onde o uso do subíndice 1 será transparente em breve); para $a=$ $a_{c}=1.401155189092 \ldots$ temos o chamado ponto de Feigenbaum (ou ponto de Feigenbaum-Coullet-Tresser) e corresponde ao limiar do caos, ou ainda caos fraco, com expoente de Lyapunov $\lambda_{1}\left(a_{c}\right)=0$. A sensitividade às condições iniciais $\xi \equiv \lim _{\Delta x_{0} \rightarrow 0} \frac{\Delta x_{t}}{\Delta x_{0}}$ satisfaz $\xi(t)=e^{\lambda_{1}(a) t}$ quando $\lambda_{1}(a)>0$, e satisfaz $\xi(t)=e_{q_{s e n}}^{\lambda_{q_{s e n}}(a) t}$ quando $\lambda_{1}(a)=0$, onde $q_{\text {sen }}=$ $0.244487701341282066198 \ldots$ (sen indica sensitividade), e $\lambda_{q_{s e n}}\left(a_{c}\right)=1 /\left(1-q_{s e n}\right)>0$ [27-29].

Para caracterizar o ritmo de crescimento da entropia com o tempo quando o sistema encontra-se inicialmente em uma diminuta região do espaço de fase (neste caso $x$ ) e passa a explorar o espaço de fase todo, define-se a produção de entropia por unidade de tempo $K_{B G} \equiv$ $\lim _{t \rightarrow \infty} \frac{S_{B G}(t)}{t}$. A identidade de Pesin garante $K_{B G}=$ $\lambda_{1}(a)>0$ para todo valor de $a$ correspondendo a caos forte. Mas esta identidade implica $0=0$ para $a=a_{c}$. Isto é sem dúvida correto porém pouco informativo. A entropia $S_{q}$ permite uma identidade mais geral e interessante, qual seja

$$
K_{q_{s e n}} \equiv \lim _{t \rightarrow \infty} \frac{S_{q_{s e n}}(t)}{t}=\lambda_{q_{s e n}}>0 .
$$

Tanto para caos forte e caos fraco temos que, para $q>$ $q_{\text {sen }}, K_{q}=0$ (que inclui em particular o resultado $0=0$ da identidade de Pesin; para $q<q_{\text {sen }}$ temos $K_{q} \rightarrow \infty$; somente para $q=q_{\text {sen }}$ temos $K_{q}$ finito. A identidade tradicional de Pesin corresponde assim ao caso particular $q_{\text {sen }}=1$.

Podemos verificar ainda uma outra propriedade notável, ligada à versão dinâmica do Teorema do Limite Central. Se somamos os valores de $x_{t}$ para $N$ valores consecutivos e escalamos adequadamente, obtemos, no limite $N \rightarrow \infty$, um atrator Gaussiano quando $\lambda_{1}(a)>0$, e um atrator $q_{\text {attract }}$-Gaussiano com $q_{\text {attract }}=1.66 \pm 0.05$ quando $\lambda_{1}(a)=0$, em particular para $a=a_{c}$. Ver Fig. 2

\subsection{Mapa standard}

O mapa padrão (ou mapa standard, ou ainda de TaylorChirikov) é definido como segue:

$$
\begin{aligned}
& p_{n+1}=p_{n}-K \sin \left(x_{n}\right)(\bmod 2 \pi) \\
& x_{n+1}=x_{n}+p_{n+1}(\bmod 2 \pi)
\end{aligned}
$$

onde $K$ é um parâmetro que controla o grau de não linearidade do sistema. Este mapa é útil para diversas situações físicas, e constitui um exemplo paradigmático de mapa conservativo. Exemplos típicos de ocupação do espaço de fases estão indicados na Fig. 3 É evidente que os casos $K=0$ e $K=8$ são extremos no sentido que o espaço de fases consiste somente de ilhas de estabilidade no primeiro exemplo e exibe um mar caótico no segundo. Os outros dois exemplos ilustram como o mar caótico emerge quando $K$ cresce de zero para valores mais altos 31.

Sempre ao longo das linhas do Teorema do Limite Central, somamos $N$ sucessivos valores da variável $x$ após centralização. Escalando adequadamente a variável soma, verificamos numericamente que o histograma se aproxima, para $N>>1$, a uma $q_{\text {attract }}$-Gaussiana com $q \simeq 2$. No cálculo numérico $\left[31,32\right.$ foi obtido $q_{\text {attract }}=$ 1.935 com uma pequena barra de erro (ver Fig. 4), mas recentemente o resultado analítico foi atingido e ele 

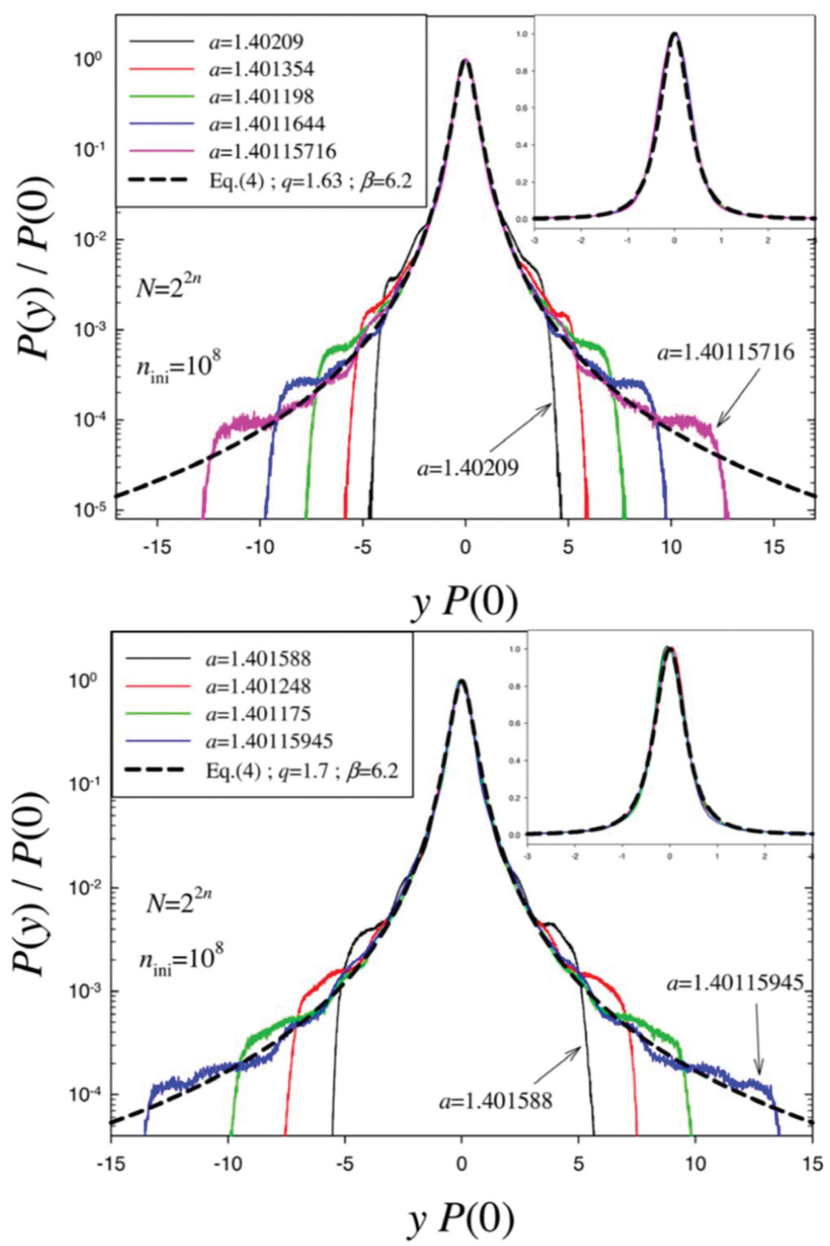

Figura 2: Colapso das densidades de probabilidades para os casos $N=2^{2 n}$, onde $2 n$ é impar (topo), ou par (fundo). Quando $n$ cresce, o histograma se aproxima gradualmente, para regiões crescentemente largas, de uma $q_{\text {attract }}$-Gaussiana $P(y) / P(0)=e_{q_{\text {attract }}}^{-\beta[y P(0)]^{2}}$ com $\left(q_{\text {attract }}, \beta\right) \simeq(1.66,6.2)$. Inserção: Representação linear-linear dos dados para uma melhor visualização da região central. Detalhes em [30].

fornece $q_{\text {attract }}=2$, i.e., uma distribuição de CauchyLorentz 33 .

\subsection{Sistemas Hamiltonianos clássicos}

Vários sistemas clássicos Hamiltonianos de $N$ corpos com os graus de liberdade configuracionais localizados em rede $d$-dimensional tem sido estudados; mais especificamente formas generalizadas do ferromagneto inercial $\mathrm{XY}$, do ferromagneto inercial de Heisenberg, e do sistema de osciladores anarmônicos acoplados de Fermi-PastaUlam. O Hamiltoniano é genericamente desta forma:

$$
\mathcal{H}_{N}=K_{N}+V_{N}=K_{N}+\sum_{i j} V_{i j}(i, j=1,2,3, \ldots, N),
$$

onde $K_{N}$ é a energia cinética total e $V_{i j} \propto 1 / r_{i j}^{\alpha}$ $(\alpha \geq 0)$; para $d=1, r_{i j}=1,2,3 \ldots$; para $d=2$, $r_{i j}=1, \sqrt{2}, 2, \ldots$; para $d=3, r_{i j}=1, \sqrt{2}, \sqrt{3}, 2, \ldots$, com $N>>1$. No limite $\alpha \rightarrow \infty$ as únicas interações existentes são entre sítios primeiros vizinhos, i.e., para $r_{i j}=1$ (consequentemente $V_{N} \propto N$ ); para $\alpha=0$, existem interações de igual intensidade entre todos os sítios e todos os outros da rede (consequentemente $V_{N} \propto N^{2}$ ); no caso geral temos $V_{N} \propto N \frac{N^{1-\alpha / d}-\alpha / d}{1-\alpha / d}$ 34]. Os casos $0<\alpha / d<1(\alpha / d>1)$ são respectivamente referidos como interações de longo (curto) alcance. Resultados numéricos de primeiros princípios (dinâmica molecular, usando exclusivamente a lei de Newton) destes sistemas estão ilustrados na Fig. 5 para o modelo $\alpha$-XY. Vemos que as célebres 'impressões digitais' da MEBG, quais sejam a distribuição Maxwelliana de velocidades e o fator de BG para as energias, são substituídos por $q$ Gaussianas e $q$-exponenciais no regime preponderadamente de longo alcance.

Verificamos, entretanto, que acima do valor crítico $\alpha / d=1$ uma região intermediária existe para a qual observamos numericamente $q>1$. No presente estágio não pode ser excluido que isto não seja uma consequência da finitude $N$ do tamanho do sistema e/ou do intervalo dentro do qual a média temporal é realizada e/ou do tempo transcorrido antes de iniciar a média temporal. Somente resultados analíticos (até agora inatingíveis) ou cálculos numéricos extremamente pesados poderiam esclarecer definitivamente esta região complexa. Poderia, por exemplo, acontecer que resultados relevantes não triviais requeiram simultaneamente $N \rightarrow \infty$ and $t \rightarrow \infty$ ao longo de caminhos apropriadamente escalados. Tal foi o caso efetivamente verificado no modelo $\alpha$-Fermi-PastaUlam, como ilustrado na Fig. 6.

\subsection{Sistemas confinados de muitos corpos na presença de super-amortecimento}

Vários sistemas confinados de muitos corpos com graus de liberdade translacionais existem cuja evolução acontece em presença de super-amortecimento, portanto sem componente inercial. Podemos citar turbulência de vórtices em supercondutores do tipo II acoplados por interações repulsivas de curto alcance [37], assim como outros semelhantes $d$-dimensionais com acoplamentos repulsivos em lei de potencia do tipo $1 / r^{\alpha}(\alpha / d>1)$ [38, eletrólitos 39], citando somente alguns. Para a distribuição estacionária de posições (e também das velocidades ao longo do tempo) foi encontrado (analítica assim como numericamente com dinâmica molecular) $q_{\text {attract }}=0$ e $q_{\text {attract }}=1-\alpha / d$, respectivamente. $\mathrm{Na}$ Fig. 7 ilustramos o exemplo dos supercondutores do tipo II. Em vários sistemas deste tipo tem-se verificado analiticamente o Princípio Zero da Termodinâmica, o Teorema H, a $q$-invariância da eficiência dos ciclos de Carnot, obtendo também as equações de estado, ilustrando assim um fato fisicamente muito relevante, qual seja que a MEBG é suficiente mas não necessária para a validade da Termodinâmica clássica, refinando assim um paradigma velho de uns 140 anos. 

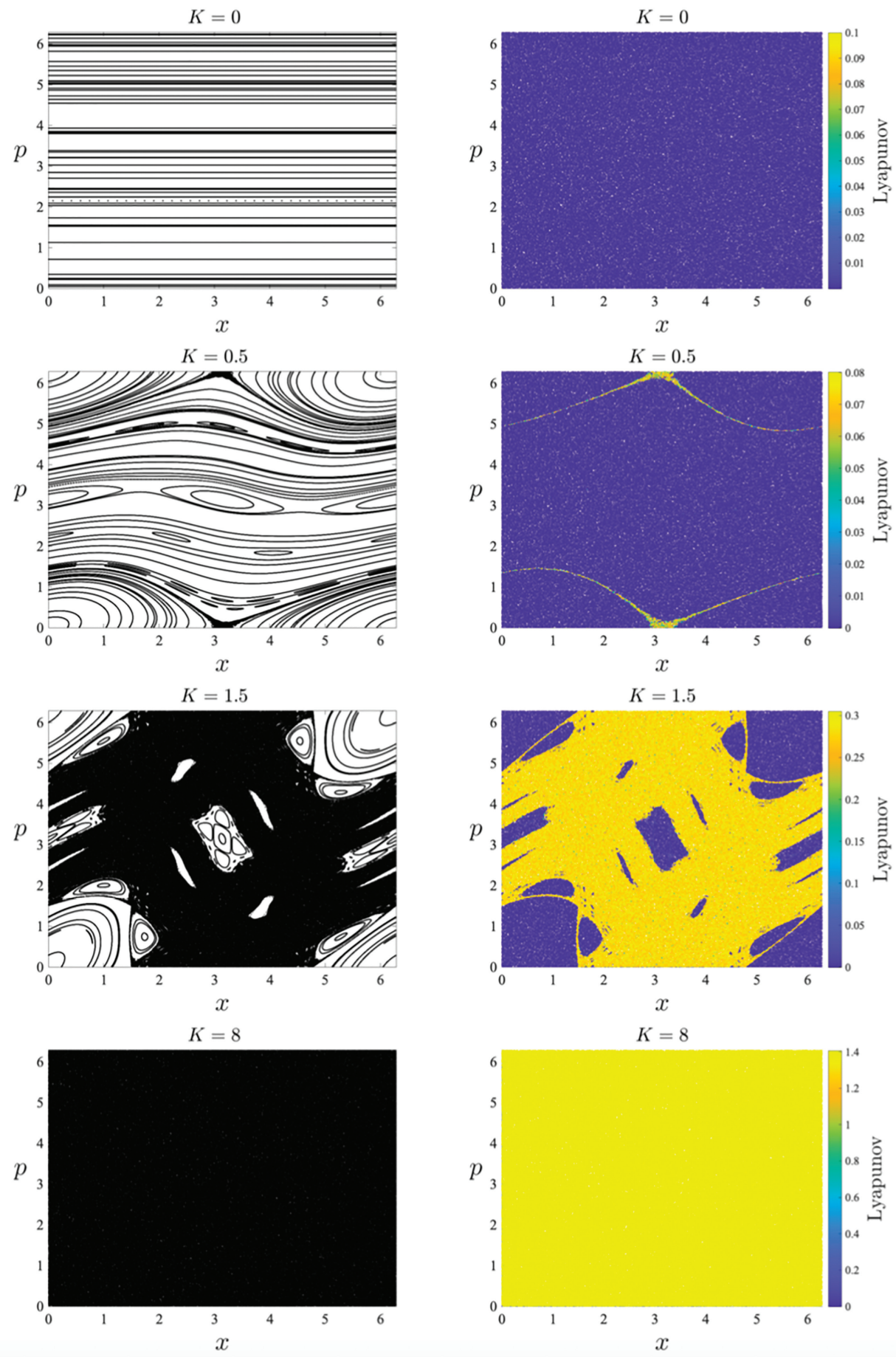

Figura 3: Mapa standard para valores representativos do parâmetro K. Esquerda: Ocupação no espaço de fases. Direita: Expoente de Lyapunov. Os exemplos $K=0$ e $K=8$ ilustram respectivamente os domínios de validade da $q$-estatística e da estatística de BG; os exemplos $K=0.5$ e $K=1.5$ ilustram situações intermediárias. Detalhes em [31].

\subsection{Transição de fases quântica}

Os sistemas quânticos de muitos corpos podem apresentar transições de fase a $T=0$. Tal é o caso, por exemplo, da cadeia de Ising com interações ferromagnéticas de primeiros vizinhos, na presença de campo transverso. Existe um valor deste campo onde um fenômeno critico acontece que conecta os estados coletivos ferromagnético com o paramagnético. Neste ponto acontece um fenômeno peculiar. A entropia total do sistema de $N$ spins é zero, em conformidade com o Terceiro Princípio da Termodinâmica, visto que o sistema está num estado puro. Mas a entropia de um bloco qualquer de $L$ spins com $L<N$ é positiva, pois ela corresponde 

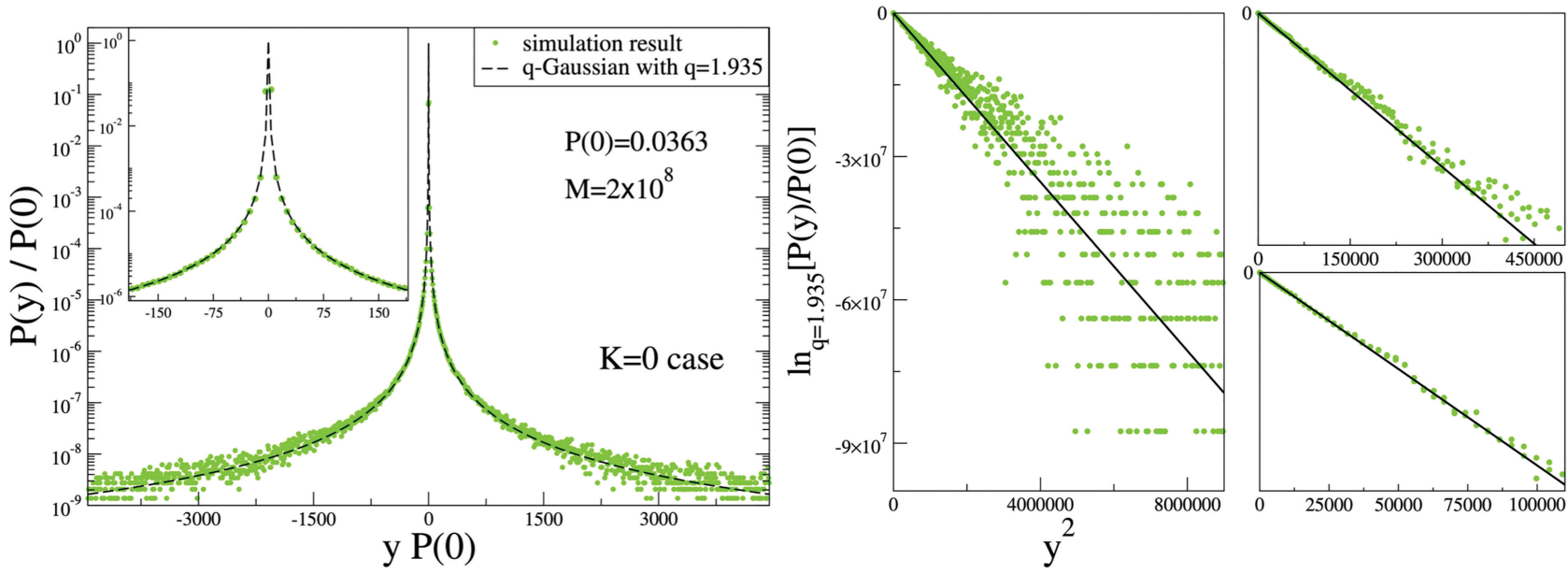

Figura 4: Distribuição de probabilidades para o caso $K=0$. Um grande número $\left(M=2 \times 10^{8}\right)$ de condições iniciais foi usado para ter uma boa estatística. O número de somandos nas simulações também é grande $\left(N=2^{22}\right)$ de modo de visualizar a distribuição limite ao longo de muitas décadas. Esquerda: representação log-linear. Direita: representação q-log - quadrática; as linhas retas pretas representam precisamente a distribuição $q$-Gaussiana em diferentes escalas. Detalhes em [31].
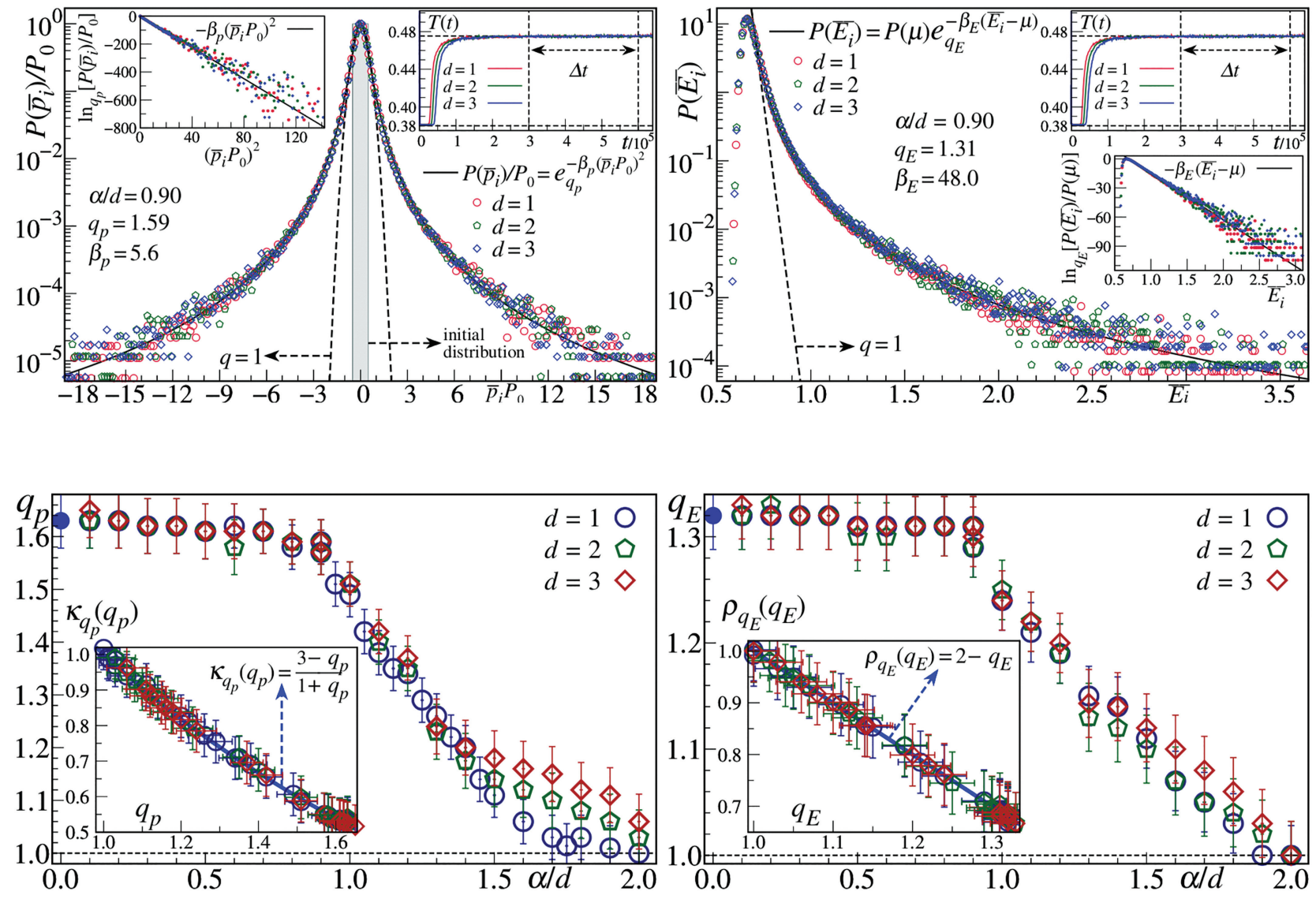

Figura 5: Ferromagneto inercial $\alpha$-XY clássico em $d$-dimensões (para $d=1,2,3$ ). As medias temporais foram feitas ao longo dos intervalos $\Delta t$ indicados nas inserções. Topo esquerda: llustração (curva preta) de $q_{p}$-Gaussiana para a distribuição dos momentos de uma partícula (para comparação, a Gaussiana de BG é mostrada em curva tracejada). Topo direita: llustração (curva preta) da $q_{E}$-exponencial para as energias de uma partícula (para comparação, o fator de BG é mostrado na curva tracejada). Fundo esquerda: A dependência em $\alpha / d$ do índice $q_{p}$ (na inserção é mostrado em curva preta o resultado analítico correspondente a $q$-Gaussianas). Fundo direita: A dependência em $\alpha / d$-dependence do índice $q_{E}$ (na inserção é mostrado em curva preta o resultado analítico correspondente a $q$-exponenciais). Detalhes em [35]. 


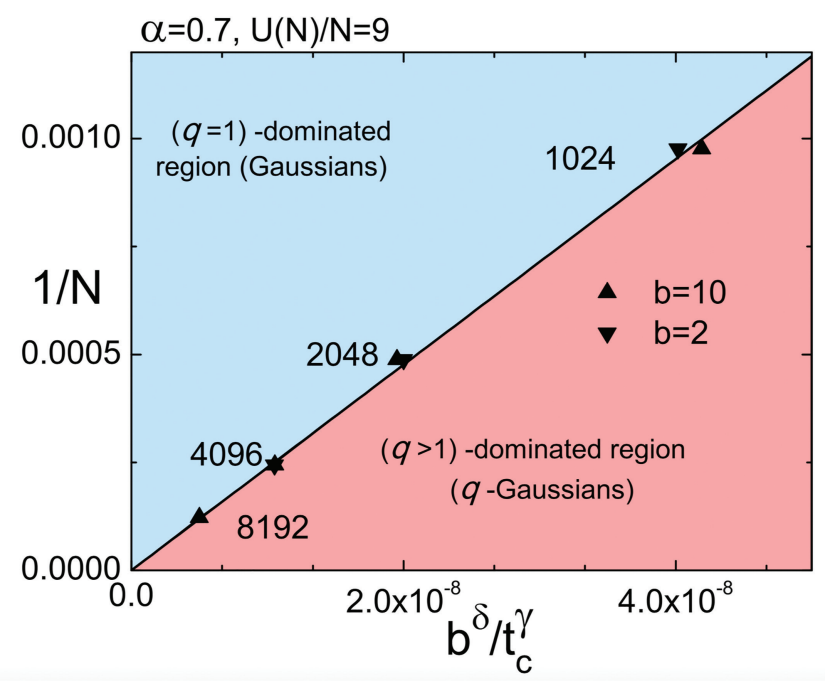

Figura 6: Visão unificada da fronteira do crossover BG - não BG do modelo $\alpha$-Fermi-Pasta-Ulam, onde $b$ é o coeficiente do acoplamento quártico. A linha reta continua (preta) é dada por $1 / N=D b^{\delta} / t_{c}^{\gamma}$, com $D=2.3818 \times 10^{4}, \delta=0.27048$, e $\gamma=$ 1.365. Detalhes em [36].

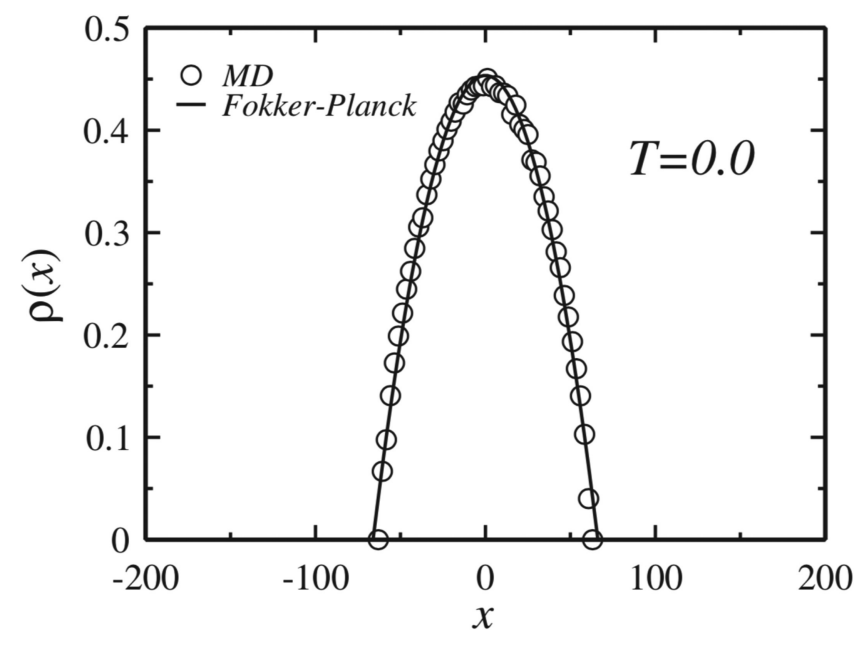

Figura 7: Perfil da densidade de partículas no estado estacionário a $T=0$, obtido por dinâmica molecular (círculos vazios), comparado com o resultado teórico ( $q_{\text {attract }}$-Gaussian com $q_{\text {attract }}=0$; linha cheia). A posição $x$ é medida em unidades do comprimento de penetração de London $\lambda$, e a densidade do estado estacionário $\rho(x)$ é expressa em unidades de $\lambda^{-2}$. Ver detalhes em [37].

a um estado quânticamente emaranhado e não mais a um estado puro. No limite $N \rightarrow \infty$ temos que, para $L \gg 1, S_{B G}(L) \propto \ln L$, o que viola a extensividade da entropia do bloco. Como consequência, a maior parte das relações termodinâmicas usuais são violadas, tornando assim inaplicável seu uso com $S_{B G}$. Entretanto, existe um valor $q_{\text {ent }} \leq 1$ tal que $S_{q_{\text {ent }}}(L) \propto L$, recuperando assim a validade da termodinâmica clássica na sua plenitude. Este valor especial do índice é dado por $q_{\text {ent }}=\frac{\sqrt{9+c^{2}}-3}{c}$ 40, onde $c$ é a chamada carga central

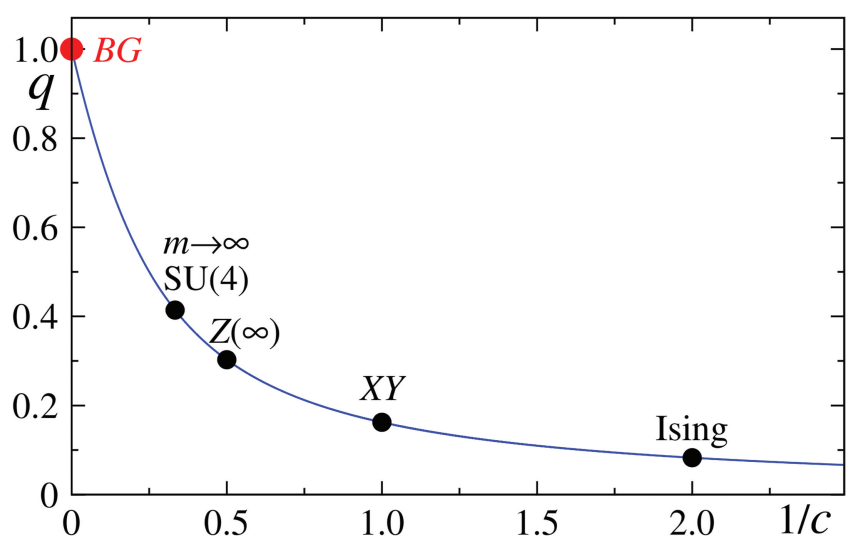

Figura 8: $O$ índice $q_{e n t}$ foi determinado 40 de primeiros princípios, mais precisamente a partir da classe de universalidade do Hamiltoniano. A carga central vale $c=1 / 2$ e $c=1$ respectivamente para as cadeias ferromagnéticas de Ising e $X Y$ na presença do campo transverso na criticalidade a $T=0$. Para outros modelos ver [41 42]. No limite $c \rightarrow \infty$ recuperamos o valor de BG, i.e., $q_{e n t}=1$. Para $c$ arbitrário, a entropia não $a d i$ tiva $S_{q_{e n t}}(L)$ do sistema é termodinâmicamente extensiva para e somente para $q_{e n t}=\frac{\sqrt{9+c^{2}}-3}{c}$ (portanto $c=\frac{6 q_{e n t}}{1-q_{e n t}^{2}}$; para $c=4$ temos $q_{\text {ent }}=1 / 2$, e para $c=6$ temos $q_{\text {ent }}=\frac{2}{\sqrt{5}+1}=\frac{1}{\Phi}$, onde $\Phi$ é a razão áurea. Enfatizemos que este valor anômalo de $q_{\text {ent }}$ acontece somente no ponto crítico quântico de segunda ordem à temperatura zero; em qualquer outra situação vale o índice usual de BG para interações de curto alcance, ou seja $q_{\text {ent }}=1$. Detalhes em 43].

do sistema (a classe de universalidade do ferromagneto de Ising com interações de curto alcance corresponde a $c=1 / 2)$ : ver Fig. 8

\subsection{Matéria granular}

$\mathrm{Na}$ área de baixas energias, merecem destaque especial experimentos feitos com matéria granular em Grenoble. A motivação remonta a 1995, quando Plastino e Plastino 44 mostraram que a chamada Equação dos Meios Porosos (Porous Medium Equation), na presença de um potential confinante, está conectada à entropia $S_{q}$ e à distribuição que a otimiza, a $q$-Gaussiana (proportional a $\left.e_{q}^{-\beta_{q} x^{2}}\right)$. A conexão é de fato fundamental, mais precisamente que a distribuição no estado estacionário da equação de Fokker-Planck não linear coincide com a distribuição que extremiza a entropia $S_{q}$ sob os correspondentes vínculos, e satisfaz também um teorema $H$, para todos os valores de $q$ 45. Nesta base foi posteriormente estabelecida [46] uma nova relação de escala, qual seja $\alpha=2 /(3-q)$, onde o expoente $\alpha$ de difusão anômala definido através do deslocamento quadrático $x^{2}$ escala com $t^{\alpha}$ (observe que $q=1$ fornece o expoente de movimento Browniano clássico $\alpha=1$ ). Confirmações preliminares emergiram no estudo do movimento de células de Hydra 47] e na aproximação computacional do modelo XY com interações de longo alcance [48. Mas a 

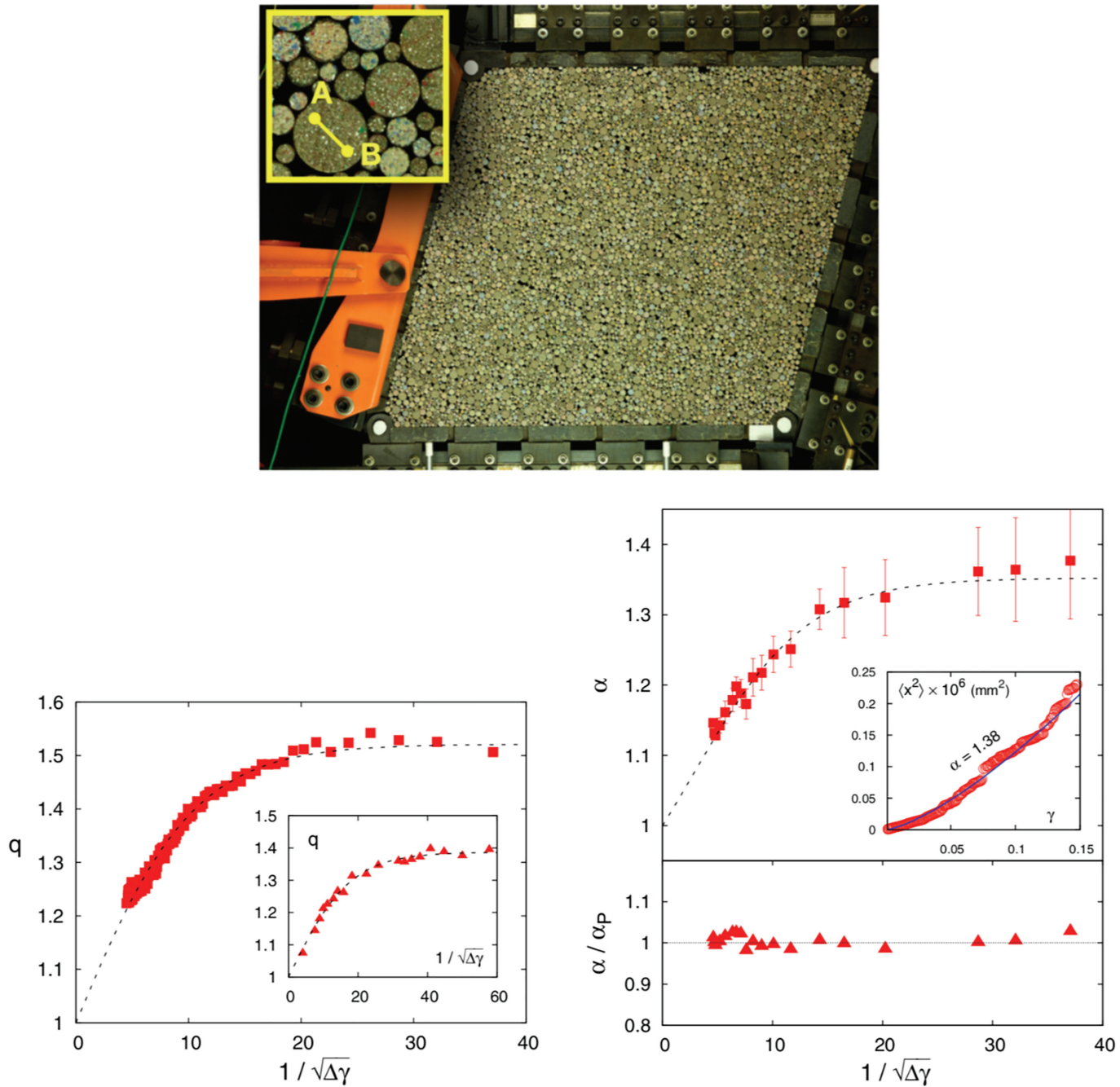

Figura 9: Verificação experimental em matéria granular da relação de escala predita em 1996 [46]. Topo: Tipo de aparelho usado (ver [49]); Fundo esquerda: Dependência do índice $q$ da distribuição $q$-Gaussiana da distribuição das flutuações num intervalo largo do parâmetro experimental $1 / \sqrt{\Delta \gamma}$; Fundo direita: Dependência do expoente $\alpha$ da difusão anômala $\left(x^{2}\right.$ escala com $\left.t^{\alpha}\right)$ com o mesmo parâmetro experimental, e verificação, com $2 \%$ de barra de erro, da predição de 1996 de $\alpha=\alpha_{P} \equiv 2 /(3-q)$ [46]. Observe que, na extrapolação $1 / \sqrt{\Delta \gamma} \rightarrow 0$, os valores de BG $(q, \alpha)=(1,1)$ emergem. Ver detalhes em [50].

validação experimental desta lei de escala num intervalo físico amplo, em matéria granular de fato [50], somente chegou 19 anos depois de sua predição: ver Fig. 9

\section{7. Átomos frios}

Outra aplicação de baixas energias concerne o movimento de átomos frios em redes óticas dissipativas. Lutz sugeriu em 2003 [51] que, sob condições apropriadas (envolvendo um mecanismo de amortecimento, e.g., o chamado resfriamento de Sísifo), a distribuição de velocidades dos átomos não seria Maxwelliana mas uma $q$-Gaussiana com $q=1+44 \frac{E_{r}}{U_{0}}$, onde $E_{r}$ é a energia atômica de recuo e $U_{0}$ é a amplitude do potencial periódico produzido pelo campo do laser. Três anos depois sua predição foi confirmada computacionalmente (Monte Carlo quântico) e experimentalmente (com átomos de Cs) [52]: ver Fig. 10.

\subsection{Altas energias na Terra}

Centenas de publicações testemunham [26] a adequação da $q$-estatística para descrever resultados das colisões em altas energias de partículas (e.g., $p p, p \bar{p}, \mathrm{Au}+\mathrm{Au}$, $\mathrm{Cu}+\mathrm{Cu}, \mathrm{Pb}+\mathrm{Pb}$ ) no LHC/CERN (detetores ALICE, CMS, ATLAS, LHCb) e no RHIC/Brookhaven (detetores BRAHMS, STAR, PHENIX). Uma ilustração é apresentada na Fig. 11

\subsection{Altas energias no espaço extraterrestre}

Observações de altas energias no espaço extraterrestre, em particular no experimento AMS (Alpha Magnetic Spectrometer), também tem sido ajustadas satisfatoriamente no quadro da $q$-estatística. Ver Fig. 12 para as distribuições de electrons e positrons, e as Figs. 13 and 14 para o fluxo de varias espécies de raios cósmicos. 

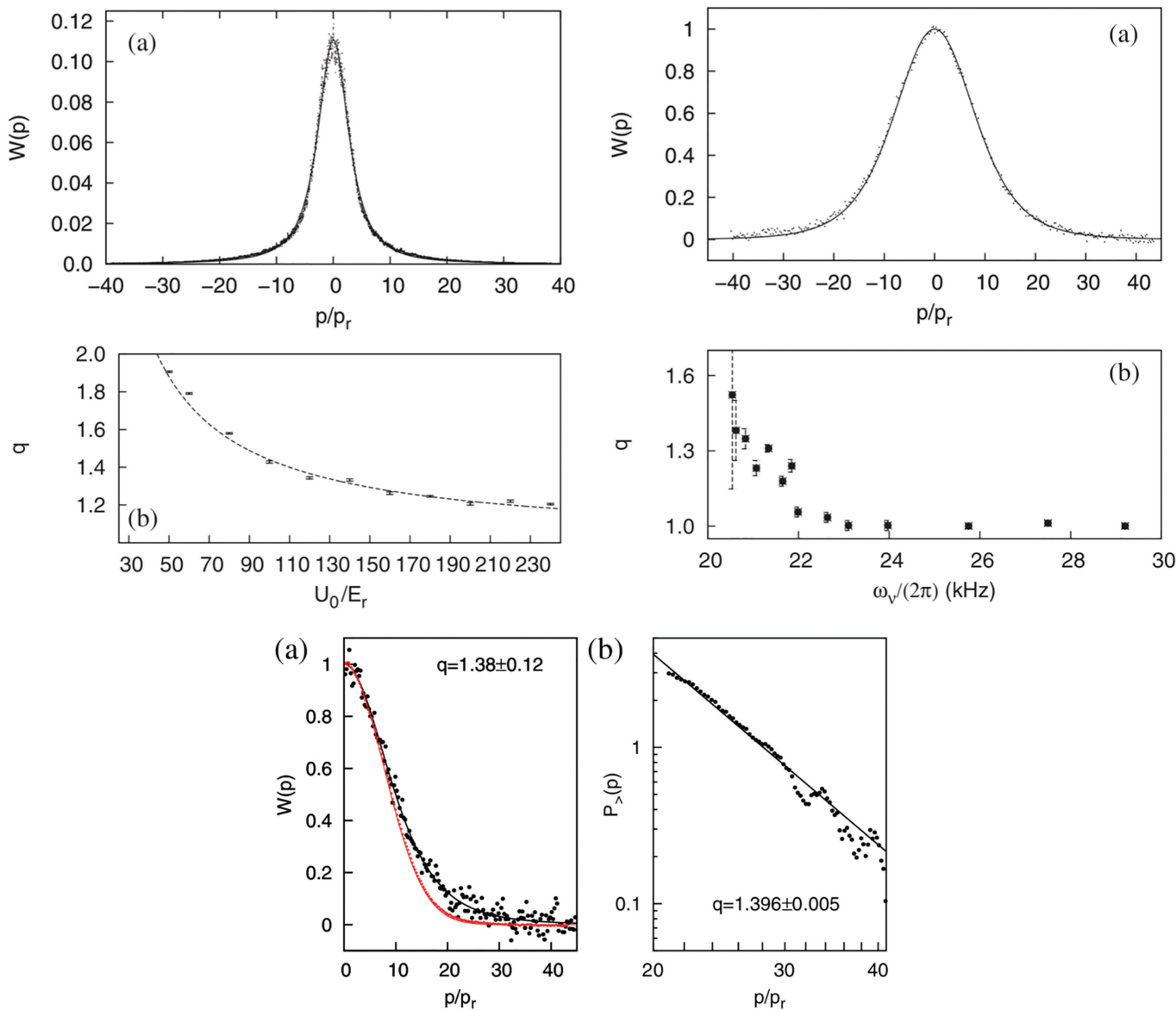

Figura 10: Verificação experimental e computacional da predição de Lutz de 2003 para átomos frios [51]. Topo esquerda: Simulações Monte Carlo quântico para a distribuição de momentos em rede unidimensional (dados pontuais mediados sobre $10^{4}$ trajetórias atômicas). Cada trajetória atómica é iniciada no estado fundamental de um dado poço. A profundidade de rede é $U_{0}=60 E_{r}$. Topo direita: Melhor ajuste experimental com $q$-Gaussiana $\left(q=1.791 \pm 0.004\right.$; ajuste $\left.R^{2}=0.995\right)$. Meio esquerda: Valores de $q$ em função da profundidade do potencial ótico. Os pontos provem de simulações Monte Carlo quânticas; a linha representa a predição $q=1+44 E_{r} / U_{0}$ [51]. Meio direita: Valores de $q$ em função da frequência vibracional no fundo do poço, obtidos ajustando com $q$-Gaussianas os experimentos. Fundo esquerda: Resultados experimentais da distribuição em rede tridimensional (pontos) e seu melhor ajuste $q$-Gaussiano (linha solida). O valor $q=1.310 \pm 0.015$ é obtido ajustando somente a parte direita da distribuição de momentos (ajuste $\left.R^{2}=0.9985\right)$. O parâmetro da rede ótica é $\omega_{v} /(2 \pi)=20.8 \mathrm{kHz}$. O máximo da distribuição é normalizado à unidade. Resultados experimentais da distribuição (pontos pretos) e seu melhor ajuste $q$-Gaussiano (linha preta sólida). O valor de $q$ está indicado na figura (ajuste $\left.R^{2}=0.9985\right)$. O parâmetro da rede ótica é $\omega_{v} /(2 \pi)=27.5 \mathrm{kHz}$. Uma Gaussiana está comparativamente indicada (linha vermelha). Fundo direita: Os pontos da distribuição para altos momentos: melhor ajuste com lei de potencia (linha sólida). Detalhes em [52 53].

\subsection{Redes complexas}

Existe uma grande diversidade de redes complexas $d$-dimensionais nos sistemas naturais, artificiais e sociais, constituídas por nós e ligações entre eles. Muitas delas são qualificadas de invariantes por escala, por exibir, na sua distribuição do número de ligações por nó, um decaimento (assintótico) em lei de potência. Muitas delas (mas não todas) crescem em função do tempo a medida que novos nós se incorporam ao sistema. Cada novo nó que chega pode se ligar a um dos nós pre-existentes através da chamada ligação preferencial (preferential attachment), regida, por exemplo, por uma probabilidade (do sítio $j$ que está chegando se ligar ao sítio $i$ pre-existente na rede) $\Pi_{i j} \propto \frac{k_{i}}{r_{i j}^{\alpha_{A}}}$ onde $k_{i}$ é o número de ligações do sítio $i$, e $\alpha_{A} \geq 0$. Tais sistemas podem ser vistos como um caso particular do domínio de aplicabilidade da MENE. Ver na Fig. 15 uma ilustração típica deste fato.

\subsection{Mecânica quântica não linear}

Existem sistemas físicos quânticos que manifestam propriedades que ultrapassam a natureza linear de equações tais como a de Schrödinger, de Klein-Gordon e de Dirac. Dispor de soluções exatas para possíveis generalizações não lineares destas equações pode ser de grande utilidade. As estruturas matemáticas que emergem 


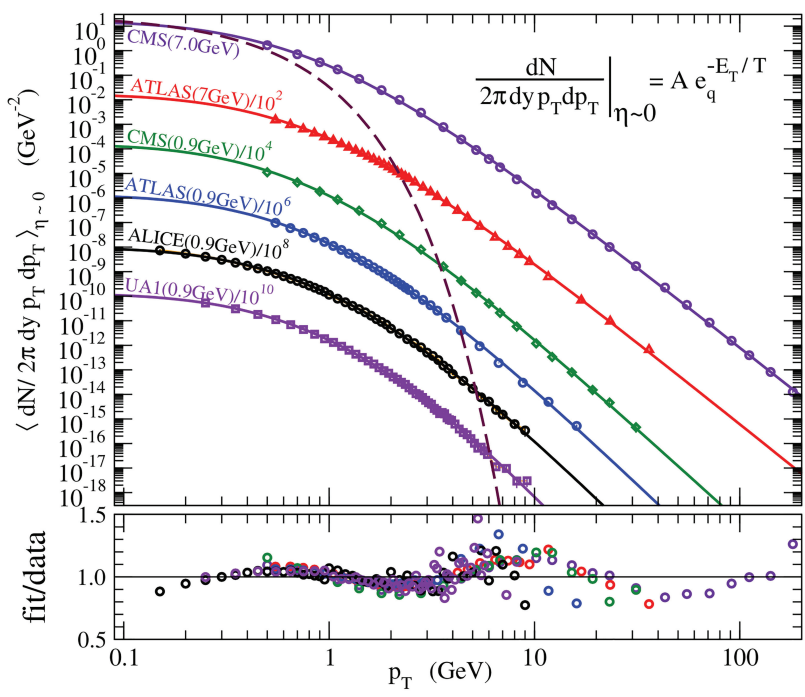

Figura 11: Comparaçao de $A e_{q}^{-E_{T} / T}$, onde $E_{T}=\sqrt{m^{2}+p_{T}^{2}}$, com distribuições experimentais de momentos transversos em colisões $p p$ collisions para rapidez central $y$. A correspondente distribuição de BG (exponencial) é ilustrada na curva tracejada. Para uma melhor visualização, tanto os dados como as curvas analíticas tem sido divididas por um fator constante como indicado. Como vemos, os ajustes são impressionantemente satisfatórios ao longo de não menos de 14 décadas na ordenada! Tal situação é praticamente sem precedentes. Com efeito, tantas décadas medidas num único experimento constitui um fato raro, que exibe o talento do esforço experimental envolvido. Para realisticamente apreciar isto, estas curvas podem ser comparadas, por exemplo, àquelas exibindo o 'crossover' da mecânica de Newton para a de Einstein para valores crescentemente altos do momento. Efetivamente se consideramos o caso dos protons nos raios cósmicos até a deteção na Terra das energias extremas, temos 11 décadas na ordenada entre o inicio da discrepância de relação de Einstein $E=\sqrt{m^{2} c^{4}+p^{2} c^{2}}$ com a relação usando a energia cinética clássica $E=m c^{2}+p^{2} / 2 m$, até o mais alto nível experimental relativista. A razão ajuste/dados estão mostrados no parte inferior, onde um comportamento aproximadamente log-periódico é observado por cima da $q$-exponencial. Tais curvas log-periódicas tem sido muito satisfatoriamente ajustadas introduzindo no indice $q$ uma pequena parte imaginaria (e.g., $q=1.14+i 0.03)$ [54, 55]. Detalhes em [56].

naturalmente na MENE permitem estabelecer uma conexão deste tipo 62 .

A $q$-onda plana $d$-dimensional é definida como segue:

$$
\Phi(\vec{x}, t)=\Phi_{0} \exp _{q}[i(\vec{k} \cdot \vec{x}-\omega t)] .
$$

Focalizemos agora uma generalização da equação de Schrödinger cuja solução exata para a partícula livre de masa $m$ é precisamente a $q$-onda plana. Seja

$$
i \hbar \frac{\partial}{\partial t}\left[\frac{\Phi(\vec{x}, t)}{\Phi_{0}}\right]=-\frac{1}{2-q} \frac{\hbar^{2}}{2 m} \nabla^{2}\left[\frac{\Phi(\vec{x}, t)}{\Phi_{0}}\right]^{2-q} .
$$

Observamos que a escala com $\Phi_{0}$ garante as dimensões físicas corretas de ambos lados da igualdade. Esta escala

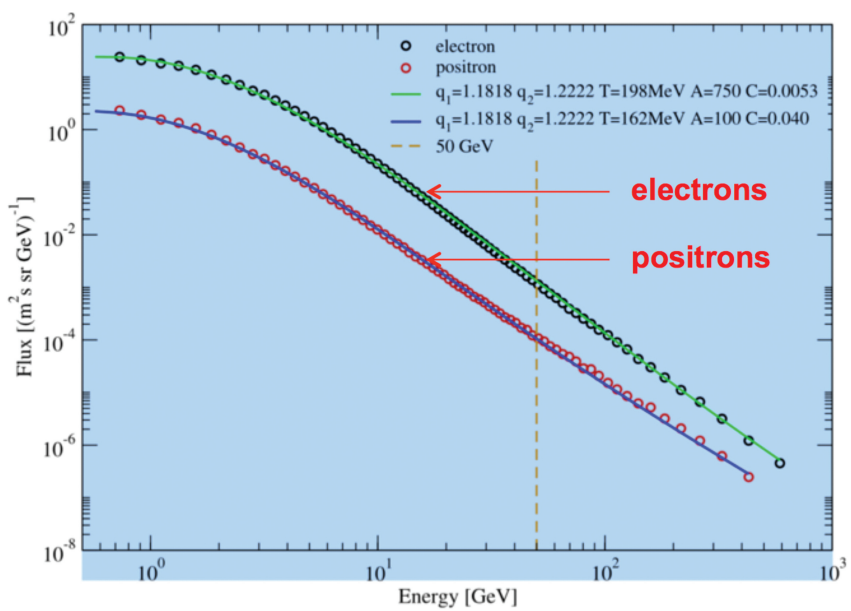

Figura 12: Os dados no AMS-02 são ajustados muito bem por combinações lineares de distribuições padrão e 'escort' com $q_{1}=$ $13 / 11=1.1818 \ldots$ e $q_{2}=1 /\left(2-q_{1}\right)=11 / 9=1.2222 \ldots$ Detalhes em [57].

se torna irrelevante somente para a equação linear, i.e., $q=1$ ]. Consistentemente, os operadores de energia e momento sao generalizados como $\hat{E}=i \hbar D_{t}$ e $\hat{p}_{n}=-i \hbar D_{x_{n}}$ respectivamente, onde $D_{u} f(u) \equiv$ $[f(u)]^{1-q} d f(u) / d u$. Estes operadores, quando atuando sobre a $q$-exponential $\exp _{q}[i(\vec{k} \cdot \vec{x}-\omega t)]$, fornecem a energia $E=\hbar \omega$ (relação de Planck) e o momento $\vec{p}=\hbar \vec{k}$ (relação de de Broglie). Considerando agora a $q$-onda plana definida na Eq. 19 e usando $\vec{k} \rightarrow \vec{p} / \hbar$ e $\omega \rightarrow E / \hbar$, verificamos que esta nova forma é solução da equação (20) com $E=p^{2} / 2 m$, para qualquer valor de $q$.

Uma estrutura totalmente similar é verificada ao $q$-generalizar consistentemente as equações quânticas relativistas de Klein-Gordon e de Dirac. As $q$-ondas planas são mais uma vez as soluções para partícula livre, satisfazendo em ambos casos a relação de Einstein $E=\sqrt{m^{2} c^{4}+p^{2} c^{2}}$, para qualquer valor de $q$ 62. Esta linha de pesquisa, que pode em tese ter utilidade no estudo da matéria escura e da energia escura, é atualmente muito ativa [26].

\subsection{Sistemas biológicos}

Um interessante estudo da migração coletiva de células (fenômeno crucial para cura de feridas, crescimento de câncer, morfogênese) é descrito em [63. As distribuições de velocidades obtidas para uma camada multicelular de rim canino de Madin Darby (MDCK) estão exibidas na Fig. 16. Elas sugerem $q$-Gaussianas com $q=1.183 \pm$ 0.035. Resultados muito semelhantes são obtidos para células do endótelio de veia umbilical humana (HUVEC) com $q=1.208 \pm 0.025$, para mioblastos de rato $\mathrm{C} 2 \mathrm{C} 12$ com $q=1.184 \pm 0.014$, e para fibroblasto de embrião de rato $\mathrm{NIH}-3 \mathrm{~T} 3 \operatorname{com} q=1.176 \pm 0.024$. 

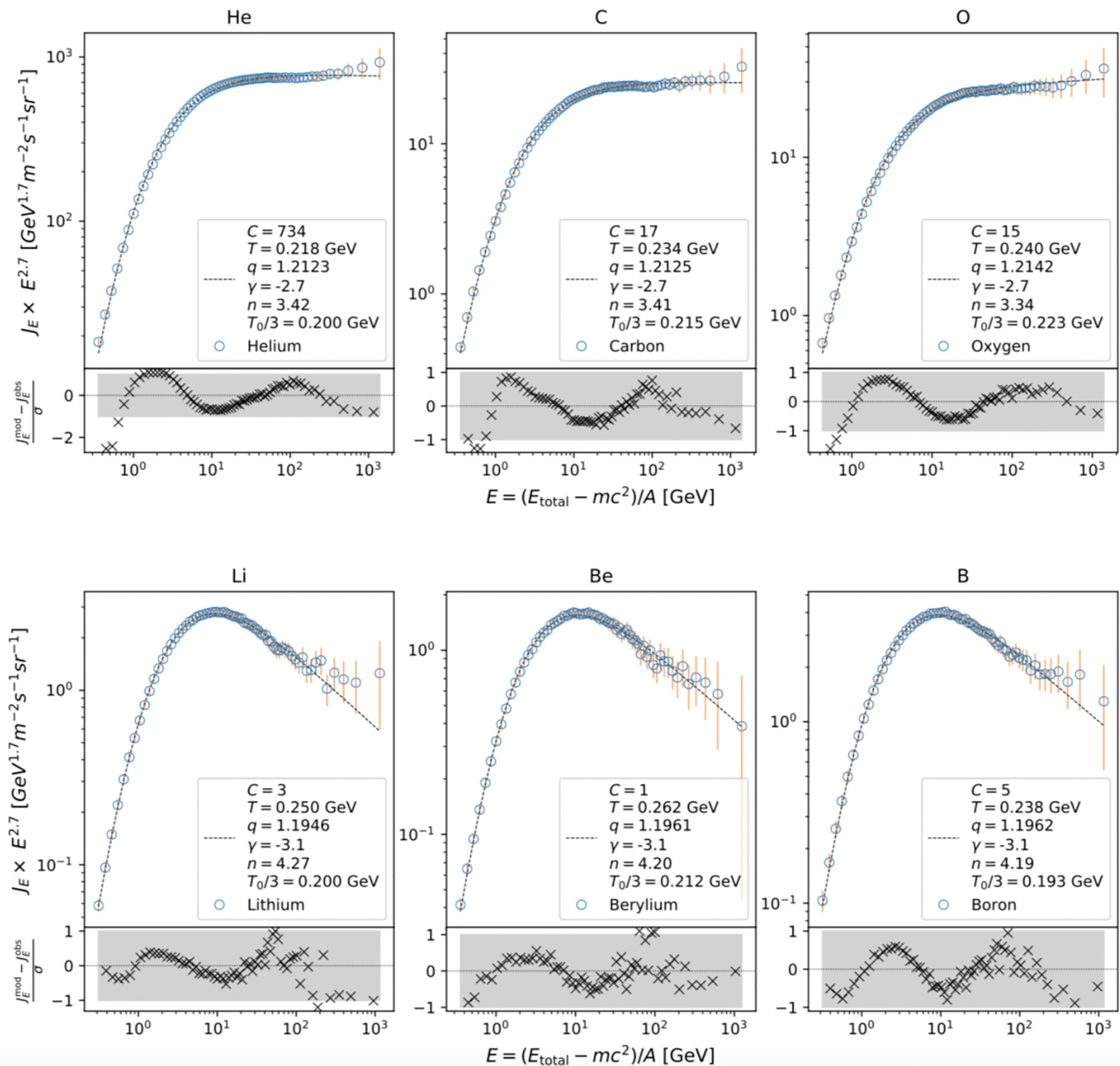

Figura 13: O fluxo de partículas de cada espécie de raios cósmicos está ajustado com $J_{E}^{\text {mod }}(E)=C E(E+2 u) e_{q}^{-E / T}$ usando três parâmetros $(C, T, q)$; a massa em $m$ repouso satisfaz $m=A u$, onde $A$ é a massa atómica e $u=0.931 G e V$ é a unidade de massa. Nesta representação log-log, o eixo vertical está multiplicado por $E^{2.7}$ para melhor visibilidade. A precisão do ajuste pode ser quantificada pelo desvio do fluxo teórico $J_{E}^{m o d}$ ao fluxo observado $J_{E}^{\text {obs }}$ em relação ao respectivo erro de medição $\sigma$. Evidentemente, quase todos os pontos observados caem dentro da barra de incerteza $\pm \sigma$ ilustrada como área cinza. A temperatura média $T_{0}$ está definida por $T_{0}=T /(4-3 q)$. A amplitude $C$ tem dimensões $[C]=\left[m^{-2} s r^{-1} s^{-1} G e V^{-3}\right]$. Detalhes em [58].

\subsection{Economia}

Existe profusa literatura sobre aplicações de $q$-estatística em economia: ver 64 e referencias ali indicadas. A título de ilustração mencionemos que Borland generalizou 65] com sucesso a equação de Black-Scholes, obtendo procedimentos que ajustam bem a realidade financeira. Ver Fig. 17

\subsection{Processamento de imagens}

Um grande número de algoritmos existem para processar, em diversos sentidos, imagens (e sinais). Não é esta a ocasião para descrever aqueles que usam, com benefício, os conceitos da MENE. Nos restringimos a apresentar aqui um que encontrou aplicação em diagnósticos importantes, quais sejam de câncer de mama 66. Ver Fig. 18

\subsection{Epidemiologia}

A pandemia do COVID sendo um sistema tipicamente complexo, é natural que se tente alguma conexão com a $q$-estatística. No trabalho [67, foi proposta heuristicamente uma expressão analítica para a evolução temporal do número de casos ativos em um pais dado. A inspiração veio de finanças e o cenário conjeturado para o pico (único, nos casos mais simples) incorporava 


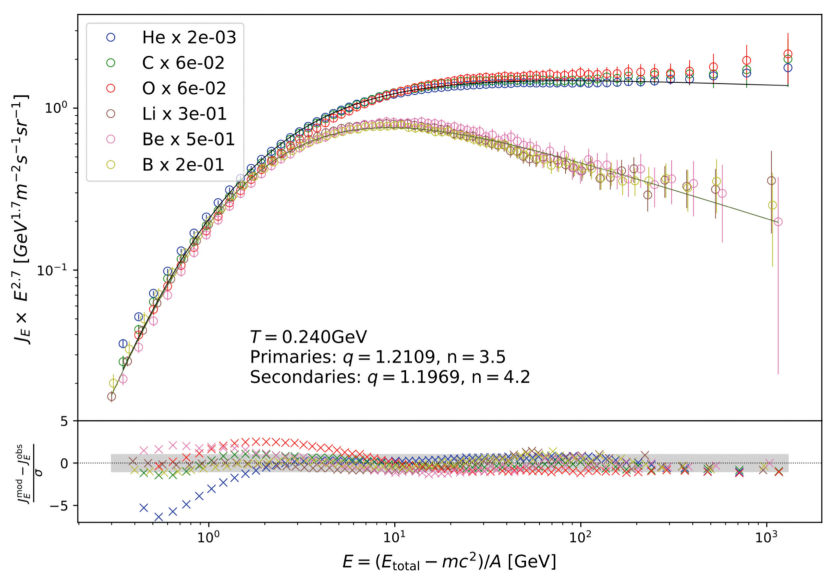

Figura 14: Cada fluxo de partículas está re-escalado com um fator conveniente tal que todos os dados aproximadamente colapsam numa única curva na região de baixas energias e as propriedades universais dos espectros dos núcleos dos raios cósmicos primários e secundários tornam-se visíveis. Para energias mais altas o espectro bifurca em primários e secundários que podem ser distinguidos através de um único parâmetro, o índice $q$, que pode ser interpretado em termos dos graus de liberdade efetivos subjacentes. Detalhes em [58].

uma subida em lei de potência, e consistentemente uma descida também em lei de potência. Segue a forma funcional proposta:

$$
\begin{aligned}
N & =C\left(t-t_{0}\right)^{\alpha} e_{q}^{-\beta\left(t-t_{0}\right)^{\gamma}} \\
& =\frac{C\left(t-t_{0}\right)^{\alpha}}{\left[1+(q-1) \beta\left(t-t_{0}\right)^{\gamma}\right]^{1 /(q-1)}},
\end{aligned}
$$

onde $C>0 ; \alpha>0 ; \beta>0 ; \gamma>0, q>1$, e $t_{0} \geq 0$. A constante $t_{0}$ indica o primeiro dia de aparição da pandemia naquele pais; convencionalmente foi escolhido $t_{0}=0$ para a China; para os outros países, $t_{0}$ corresponde ao número de dias transcorridos entre a aparição do primeiro caso na China e o primeiro caso no pais estudado. A constante de normalização $C$ reflete a população total daquele pais. Para $\alpha=0$, o valor $\gamma=1$ nos leva à $q$-exponencial; o valor $q=2$ nos leva à $q$-Gaussiana; outros valores de $\gamma$ são referidos como $q$-exponencial esticada (stretched q-exponential). Através da inspeção das influencias exercidas pelas quatro constantes não triviais $(\alpha, \beta, \gamma, q)$, tornou-se relativamente transparente que $(\alpha, \beta)$ dependem fortemente da estratégia epidemiológica implementada na região, assim como do comportamento biológico do coronavirus naquele clima geográfico. Em contraste, os parâmetros $(\gamma, q)$ parecem ter uma natureza um tanto universal, dependendo principalmente do coronavirus. Portanto, para investigar diversos países que não tinham ainda atingido seu pico, uma primeira hipótese básica foi usar os valores da China para esses parâmetros, imaginando que a China tivesse aproximadamente completado seu ciclo pandêmico. Esta hipótese de trabalho, à falta de outra melhor, forneceu resultados satisfatórios. Para os outros países, cujo pico nao tinha sido ainda atingido, usamos a mesma forma funcional (21) ajustando o resto dos parâmetros de modo a descrever adequadamente a subida em direção ao pico (ainda desconhecido). Os resultados no dia 8 de Maio de 2020 estão exibidos na Fig. 19, A aproximação que acabamos de descrever não envolve nenhum modelo epidemiológico dinâmico. Para progredir neste sentido, a q-generalização do conhecido model SEIR foi proposta também e estudada em 68.
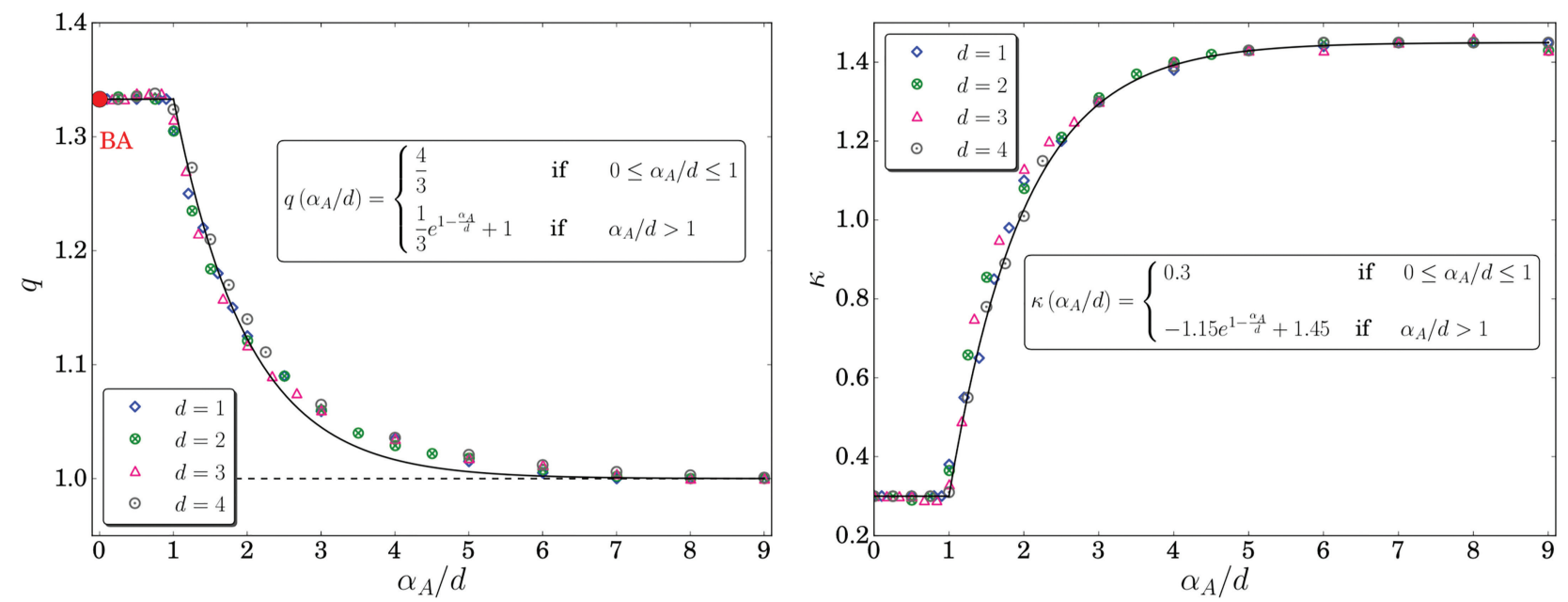

Figura 15: Redes $d$-dimensionais assintoticamente invariantes por escala (para $d=1,2,3,4$ ). Pod-se facilmente verificar que a distribuição do número $k$ de ligações por sítio é muito bem ajustada por $P(k)=P(0) e_{q}^{-k / \kappa}$. Esquerda: O índice $q$ em função de $\alpha_{A} / d$; o ponto vermelho indica a classe de universalidade de Barabási-Albert (BA) $(q=4 / 3)$ [59] 60], que é aqui recuperada com ○ o caso particular $\alpha_{A}=0$. Direita: A 'temperature' $\kappa$ (que caracteriza o número médio de ligações por nó) em função de $\alpha_{A} / d$. Em todos os casos, a descrição de BG $(q=1)$ emerge naturalmente no limite $\alpha_{A} / d \rightarrow \infty$, e mesmo antes disso. Detalhes em [61]. 
(a)

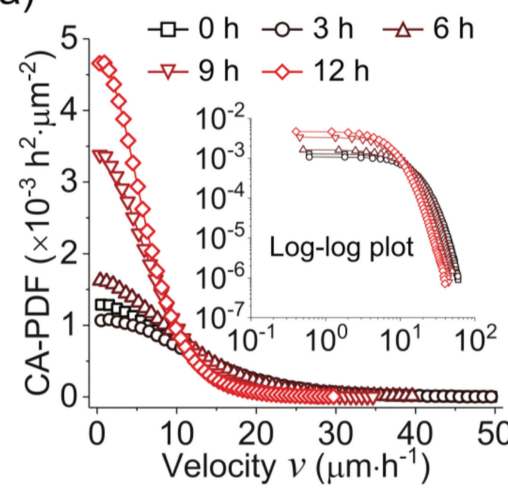

(b)

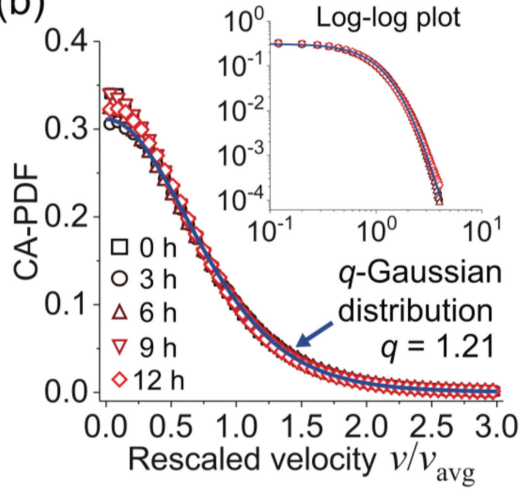

(c)

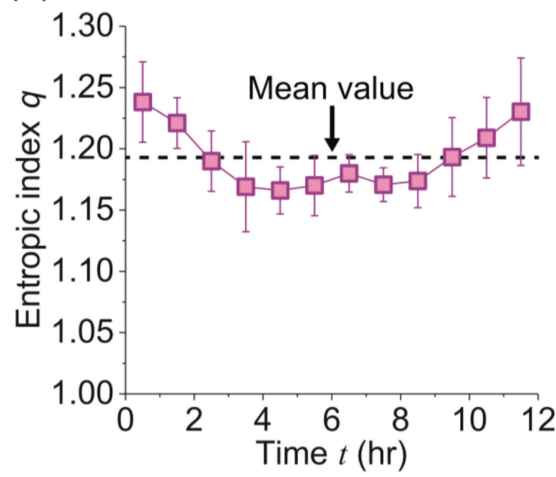

Figura 16: A distribuição de velocidades celulares (promediadas ao redor da circunferência) no MDCK é bem ajustada com uma q-Gaussiana. (a) Evolução temporal. Inserção: representação em log-log plot dos mesmos dados. (b) Colapso obtido com velocidades celulares re-escaladas a diversos tempos. Inserção: representação em log-log das distribuições. (c) Evolução temporal do índice $q$. Ver detalhes em 63 .

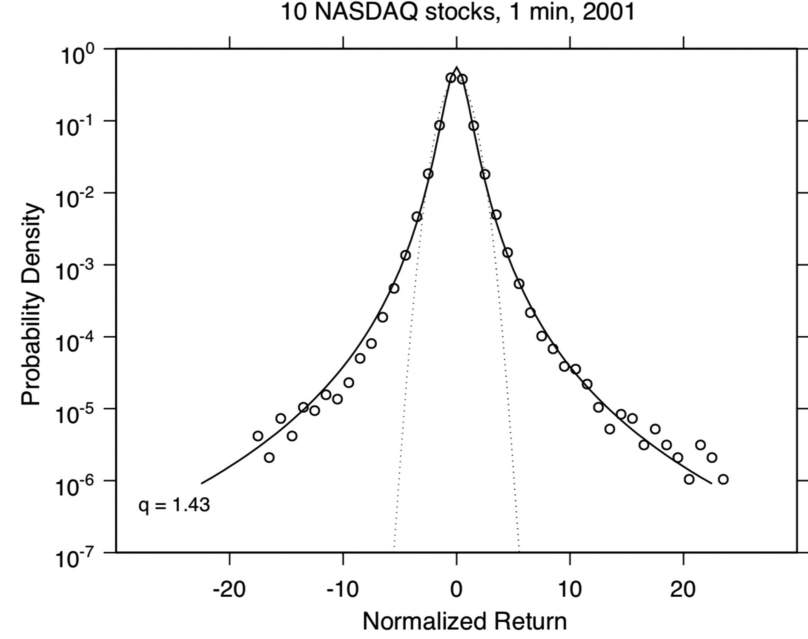

Figura 17: Distribuições de retornos logarítmicos para dez ações em intervalos de 1 minuto, normalizadas pelo desvio medio quadrático da amostra. A linha sólida corresponde a uma distribuição $q$-Gaussians distribution com $q=1.43$, que fornece um bom ajuste com os dados. Linha tracejada: modelo padrão Gaussian. Detalhes em 65].

\section{Considerações Finais}

Nestas linhas finais podemos relembrar que, nos primeiros anos após 1988, esta teoria era acolhida seja com grande entusiasmo, seja, por alguns, com grande ceticismo. No dia de hoje, pode-se verificar que mais de oito mil artigos estão disponíveis na literatura científica focalizando esta teoria [26]. Na presente revisão para a Revista Brasileira de Ensino de Física tentamos, entretanto, de nos concentrar em alguns aspectos motivacionais da teoria e de suas aplicações que esperamos possam permitir ao leitor interessado formar sua própria opinião sobre a questão, e progredir eventualmente.

Encontram-se também em [26] muitas interessantes conexões, generalizações, variações do aqui exposto,

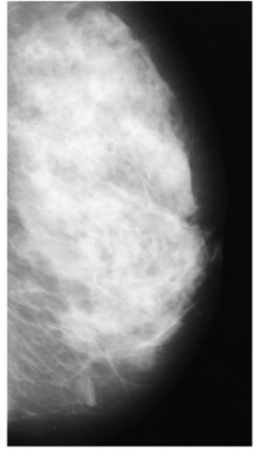

a

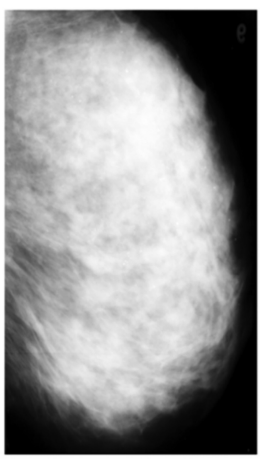

d

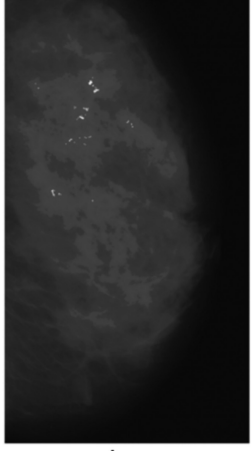

b

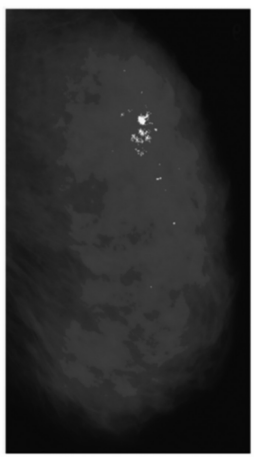

e

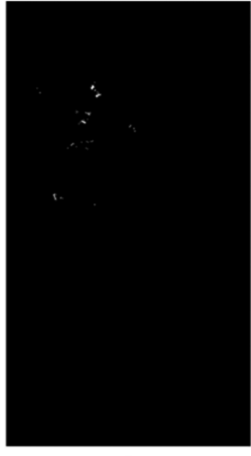

C

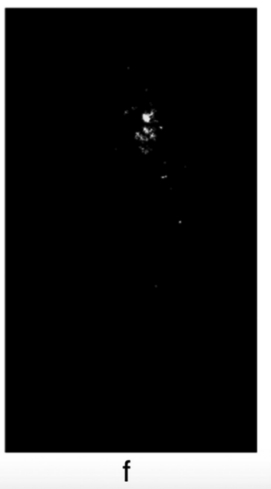

Figura 18: $O$ procedimento aqui focalizado para a deteção de microcalcificações que não usa a entropia $S_{q}$ produz resultados modestos: $80.21 \%$ Tps (verdadeiros positivos) e $8.1 \mathrm{Fps}$ (falsos positivos). Enquanto, ao introduzir a $q$-entropia, resultados auspiciosos surgem: $96.55 \%$ Tps e $0.4 \mathrm{Fps}$. Resultados do experimento: (a) mdb236, (b) imagem com as microcalcificações salientadas, (c) imagem com as microcalcificações extraídas; (d) mdb216, (e) imagem com as microcalcificações salientadas, (f) imagem com as microcalcificações extraídas. Detalhes em [66.

assim como muitas outras aplicações. É impossível revisar no atual artigo introdutório todas elas, sequer mencioná-las. Entretanto, uma linha que certamente 

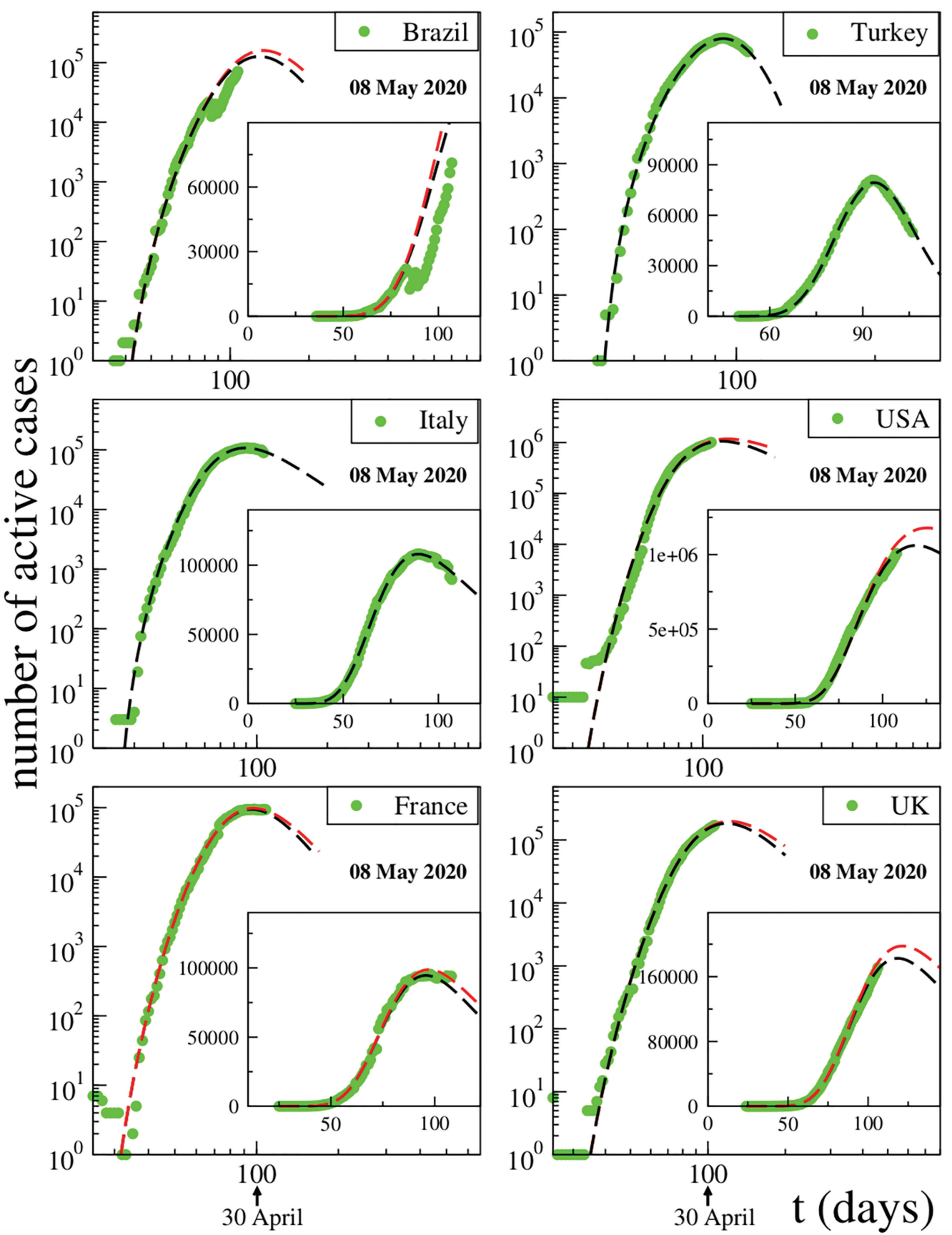

Figura 19: Ajuste dos dados de casos ativos disponíveis no 8 de Maio de 2020 para varios paises severamente afetados ao redor do mundo, com a Eq. (21). Os parâmetros de ajuste $\gamma$ e $q$ foram fixados aos valores da China toda vez que o pico não tinha sido ainda atingido. Observe que, no caso do Brasil, uma disrupção aconteceu nos dados publicamente disponíveis a meados de Abril. Desconhecemos qual seja a causa. Coincidentemente entretanto, o Presidente do Brasil decidiu de mudar seu Ministro de Saúde nesses dias. Detalhes em [67].

merece especial menção é a superestatística de BeckCohen [69, 70], diretamente relacionada com a mecânica estatística não extensiva. Grande número de problemas em aberto existe que constituem interessantes desafios. Um deles é a conceituação completa da estrutura dos $q$-tripletes e de sua conexão com as transformações de Möbius [71. A importância desta questão pode dificilmente ser exagerada pois inclui situações paradigmáticas tais como uma simples cadeia unidimensional de rotores clássicos acoplados entre primeiros vizinhos. Com efeito, em equilíbrio térmico este sistema claramente corresponde a $q=1$, mas em estado estacionário fora de equilíbrio corresponde a $q=1.55$ [72]. Eu sou eu e minha circunstância, afirmava Ortega y Gasset.

Terminemos com um toque histórico que é motivo de frequente curiosidade: por que chamá-la de mecânica 
estatística não extensiva e não, por exemplo, de mecânica estatística não aditiva? A sua aplicação mais paradigmática é constituída pelos sistemas Hamiltonianos clássicos de muitos corpos com interações de longo alcance. Neste caso, a energia interna total é termodinâmicamente não extensiva, porém a correspondente entropia total exige, presumivelmente em grande número de situações, usar formas entrópicas não aditivas de modo de garantir sua extensividade termodinâmica. Nos meandros do pensamento humano, acabou prevalecendo, para a presente generalização da mecânica estatística de Boltzmann-Gibbs, a denominação pela qual é conhecida hoje.

\section{Agradecimentos}

Agradeço calorosamente E.P. Borges por numerosos comentários decorrentes de uma leitura crítica do manuscrito, assim como aos diversos autores identificados nas várias figuras aqui reproduzidas, muitas delas fazendo parte do artigo de revisão [3]. Este trabalho foi parcialmente financiado pela Faperj e pelo CNPq.

\section{Referências}

[1] C. Tsallis, J. Stat. Phys. 52, 479 (1988).

[2] C. Tsallis, Nonextensive Statistical Mechanics Approaching a Complex World (Springer, New York, 2009).

[3] C. Tsallis, Entropy 21, 696 (2019).

[4] W. Braun e K. Hepp, Commun. Math. Phys. 56, 125 (1977).

[5] C. Jarzynski, Phys. Rev. Lett. 78, 2690 (1997).

[6] https://ui.adsabs.harvard.edu/abs/2017vico.book...75T, abstract.

[7] J.W. Gibbs, Elementary Principles in Statistical Mechanics - Developed with Especial Reference to the Rational Foundation of Thermodynamics (Charles Scribner's Sons, New York, 1902).

[8] O. Penrose, Foundations of Statistical Mechanics: A Deductive Treatment (Pergamon, Oxford, 1970).

[9] R.J.V. Santos, J. Math. Phys. 38, 4104 (1997).

[10] S. Abe, Phys. Lett. A 271, 74 (2000).

[11] P. Jizba e J. Korbel, Phys. Rev. Lett. 122, 120601 (2019).

[12] C. Tsallis, Braz. J. Phys. 29, 1 (1999).

[13] C. Tsallis e L.J.L. Cirto, Eur. Phys. J. C 73, 2487 (2013).

[14] C. Tsallis, em Nonextensive Entropy - Interdisciplinary Applications, editado por M. Gell-Mann e C. Tsallis (Oxford University Press, New York, 2004).

[15] E.M.F. Curado e C. Tsallis, J. Phys. A. 25, 1019 (1992).

[16] C. Tsallis, R.S. Mendes e A.R. Plastino, Physica A 261, 534 (1998).

[17] G.L. Ferri, S. Martinez e A. Plastino, J. Stat. Mech.Theory and Experiment P04009 (2005).

[18] C. Tsallis e Z.G. Arenas, EPJ Web of Conferences 71, 00132 (2014).
[19] S. Umarov, C. Tsallis e S. Steinberg, Milan J. Math. 76, 307 (2008).

[20] S. Umarov, C. Tsallis, M. Gell-Mann e S. Steinberg, J. Math. Phys. 51, 033502 (2010).

[21] H. Touchette, Phys. Rep. 478, 1 (2009).

[22] G. Ruiz e C. Tsallis, Phys. Lett. A 376, 2451 (2012).

[23] H. Touchette, Phys. Lett. A 377, 436 (2013).

[24] G. Ruiz e C. Tsallis, Phys. Lett. A 377, 491 (2013).

[25] U. Tirnakli, C. Tsallis e N. Ay, Approaching a large deviation theory for complex systems (2020), arxiv 2010.09508 [cond-mat.stat-mech].

[26] http://tsallis.cat.cbpf.br/biblio.htm

[27] M.L. Lyra e C. Tsallis, Phys. Rev. Lett. 80, 53 (1998).

[28] F. Baldovin e A. Robledo, Phys. Rev. E 66, R045104 (2002).

[29] F. Baldovin e A. Robledo, Phys. Rev. E 69, R045202 (2004).

[30] U. Tirnakli, C. Tsallis e C. Beck, Phys. Rev. E 79, 056209 (2009).

[31] U. Tirnakli e C. Tsallis, Nonlinear Phenomena in Complex Systems 23, 149 (2020).

[32] U. Tirnakli e E.P. Borges, Scientific Reports 6, 23644 (2016).

[33] A. Bountis, J.J.P. Veerman e F. Vivaldi, Phys. Lett. A 384, 126659 (2020).

[34] C. Anteneodo e C. Tsallis, Phys. Rev. Lett. 80, 5313 (1998).

[35] L.J.L. Cirto, A. Rodriguez, F.D. Nobre e C. Tsallis, EPL 123, 30003 (2018).

[36] H. Christodoulidi, C. Tsallis e T. Bountis, EPL 108, 40006 (2014).

[37] J.S. Andrade Jr., G.F.T. da Silva, A.A. Moreira, F.D. Nobre e E.M.F. Curado, Phys. Rev. Lett. 105, 260601 (2010).

[38] A.A. Moreira, C.M. Vieira, H.A. Carmona, J.S. Andrade Jr. e C. Tsallis, Phys. Rev. E 98, 032138 (2018).

[39] G.A. Casas, F.D. Nobre e E.M.F. Curado, EPL 126, 10005 (2019).

[40] F. Caruso e C. Tsallis, Phys. Rev. E 78, 021102 (2008).

[41] F.C. Alcaraz, J. Phys. A 20, 2511 (1987).

[42] F.C. Alcaraz e M.J. Martins, J. Phys. A 23, L1079 (1990).

[43] C. Tsallis e H.J. Haubold, EPL 110, 30005 (2015).

[44] A.R. Plastino e A. Plastino, Physica A 222, 347 (1995).

[45] V. Schwammle, F.D. Nobre e E.M.F. Curado, Phys. Rev. E 76, 041123 (2007).

[46] C. Tsallis e D.J. Bukman, Phys. Rev. E 54, R2197 (1996).

[47] A. Upadhyaya, J.P. Rieu, J.A. Glazier e Y. Sawada, Physica A 293, 549 (2001).

[48] A. Rapisarda e A. Pluchino, Europhysics News 36, 202 (2005).

[49] L. Viallon-Galiner, G. Combe, V. Richefeu e A.P.F. Atman, Entropy 20, 862 (2018).

[50] G. Combe, V. Richefeu, M. Stasiak e A.P.F. Atman, Phys. Rev. Lett. 115, 238301 (2015).

[51] E. Lutz, Phys. Rev. A 67, 051402(R) (2003).

[52] P. Douglas, S. Bergamini e F. Renzoni, Phys. Rev. Lett. 96, 110601 (2006). 
[53] E. Lutz e F. Renzoni, Nature Physics 9, 615 (2013).

[54] M. Rybczynski, G. Wilk e Z. Wlodarczyk, Eur. Phys. J. Web of Conferences 90, 01002 (2015).

[55] G. Wilk e Z. Wlodarczyk, Entropy 17, 384 (2015).

[56] C.Y. Wong, G. Wilk, L.J.L. Cirto e C. Tsallis, Phys. Rev. D 91, 114027 (2015).

[57] G.C. Yalcin e C. Beck, Scientific Reports 8, 1764 (2018).

[58] M. Smolla, B. Schafer, H. Lesch e C. Beck, New Journal Physics 22, 093002 (2020).

[59] A.L. Barabási e R. Albert, Science 286, 509 (1999).

[60] T. Emmerich, A. Bunde e S. Havlin, Phys. Rev. E 89, 062806 (2014).

[61] S.G.A. Brito, L.R. da Silva e C. Tsallis, Scientific Reports 6, 27992 (2016)

[62] F.D. Nobre, M.A. Rego-Monteiro e C. Tsallis, Phys. Rev. Lett. 106, 140601 (2011).

[63] S.Z. Lin, P.C. Chen, L.Y. Guan, Y. Shao, Y.K. Hao, Q. Li, B. Li, D.A. Weitz e X.Q. Feng, Adv. Biosys. 4, 2000065 (2020).

[64] C. Tsallis, 19, 457 (2017).

[65] L. Borland, Phys. Rev. Lett. 89, 098701 (2002).

[66] J. Mohanalin, Beenamol, P.K. Kalra e N. Kumar, Computers and Mathematics with Applications 60, 2426 (2010).

[67] C. Tsallis e U. Tirnakli, Frontiers in Physics 8, 217 (2020).

[68] U.Tirnakli e C.Tsallis, Frontiersin Physics 8, 613168 (2020).

[69] C. Beck e E.G.D. Cohen, Physica A 322, 267 (2003).

[70] E.G.D. Cohen, Pramana 64, 635 (2005).

[71] J.P. Gazeau e C. Tsallis, Entropy 21, 1155 (2019).

[72] Y. Li, N. Li, U. Tirnakli, B. Li e C. Tsallis, EPL 117, 60004 (2017) 